\title{
VARIETIES OF COMMUTATIVE SEMIGROUPS
}

\author{
ANDRZEJ KISIELEWICZ
}

\begin{abstract}
In this paper, we describe all equational theories of commutative semigroups in terms of certain well-quasi-orderings on the set of finite sequences of nonnegative integers. This description yields many old and new results on varieties of commutative semigroups. In particular, we obtain also a description of the lattice of varieties of commutative semigroups, and we give an explicit uniform solution to the word problems for free objects in all varieties of commutative semigroups.
\end{abstract}

\section{INTRODUCTION}

Varieties of semigroups have been extensively studied not only as a natural continuation of analogous investigations in the theory of groups [26], but also, more recently, because of their applications in language and automata theory (see $[6,29])$.

Special attention has been given to varieties of commutative semigroups, since in analogy with groups, one could expect that these varieties should be easier to handle. Recall that a variety of commutative (abelian) groups can be described by an additional identity $x^{m}=1$; and the lattice of these varieties is isomorphic to the natural numbers ordered by division (see also [13] for commutative monoids). Surprisingly, the lattice of varieties of commutative semigroups, $\mathscr{L}(\mathrm{Com})$, turns out to be fairly complicated.

This became clear first, when Schwabauer [36] proved that, in contrast with the lattice of all varieties of groups, $\mathscr{L}(\mathrm{Com})$ itself is nonmodular. Next, Burris and Nelson [4] proved that every finite partition lattice can be embedded into $\mathscr{L}(\mathrm{Com})$, and hence it obeys no special lattice laws. In view of the later result [31], this means that $\mathscr{L}(\mathrm{Com})$ contains all finite lattices as sublattices.

In a positive direction, Perkins [27] showed that every equational theory of commutative semigroups is finitely based, and hence $\mathscr{L}(\mathrm{Com})$ is countable and has no infinite descending chains. From a general result of Evans [8,9], it follows also that every equational theory of commutative semigroups is decidable. Yet, no explicit uniform solution to the word problems for free semigroups is known. Nelson [24] described a certain natural sublattice $\mathscr{L}_{N}(\mathrm{Com})$ of $\mathscr{L}(\mathrm{Com})$, calling it a "skeleton", and investigated relationships between

Received by the editors January 28, 1992.

1991 Mathematics Subject Classification. Primary 20M07, 20M14; Secondary 20M05, 08B05.

Key words and phrases. Commutative semigroup, variety, equational theory, lattice of varieties, identity, consequence relation.

Supported in part by the Alexander von Humboldt Stiftung. 
intervals set up by this sublattice. This extensive work was used by Almeida [2] to establish some special order properties (the so-called, better-quasi-ordering) of sublattices of $\mathscr{L}(\mathrm{Com})$. In particular, he proved that every proper sublattice of $\mathscr{L}(\mathrm{Com})$ consisting of subvarieties of a member of $\mathscr{L}(\mathrm{Com})$ has no infinite antichains. He also pointed out the central role played by the sublattice $\mathscr{L}(\mathscr{N})$ of varieties of nilpotent semigroups. The structure of the latter was investigated in [18] by Korjakov. In particular, he showed that also $\mathscr{L}(\mathscr{N})$ itself contains every finite lattice as a sublattice (which, in fact, is implicit in [4]) and therefore, in his opinion, even the complete description of this special sublattice is impossible.

Another well-behaved sublattice was described by Schwabauer [37], who proved that the sublattice $\mathscr{L}_{S}(\mathrm{Com})$ of varieties defined by identities of a certain special form is distributive. Nelson [24] gave another proof and showed that $\mathscr{L}_{S}(\mathrm{Com})$ is in fact a maximal modular sublattice in $\mathscr{L}(\mathrm{Com})$. Still another proof was provided in [2], but the structure of the Schwabauer sublattice remains unknown.

For other, more special and more detailed results see also $[1,5,14,21,22$, $23,25,33,38,40,41,42]$ and the surveys [7, 34, 35]. For descriptions of the lattices of varieties of some other comprehensive classes of semigroups see [11] and [30].

In the present paper, we describe all varieties of commutative semigroups and their lattice $\mathscr{L}(\mathrm{Com})$ in terms of certain well-quasi-ordering on the set $\Gamma$ of finite sequences of nonnegative integers. The main idea is to use known sublattices to partition $\mathscr{L}$ (Com) into smaller intervals.

Section 1 is devoted to establish terminology and recall some results we use. In $\S 2$, we construct the first partition of $\mathscr{L}(\mathrm{Com})$; in particular, we show that the Nelson sublattice $\mathscr{L}_{N}(\mathrm{Com})$ determines the shape of $\mathscr{L}(\mathrm{Com})$, since it is actually a retract of $\mathscr{L}(\mathrm{Com})$. This section is closely connected with Nelson's work [24], and some lemmas in this section could be easily inferred from the results of [24]. Yet, in order to make the paper more self-contained, easier to read, and to present some possible improvements, we provide our own complete proofs.

In $\S 3$, we give the full description of the Schwabauer sublattice $\mathscr{L}_{S}(\mathrm{Com})$ and the corresponding equational theories ( $S$-theories). We show that there is a certain natural well-quasi-ordering $\leq$ on the set $\Gamma$ of finite sequences of nonnegative integers such that $S$-theories are in one-to-one correspondence with (and are explicitly determined by) order filters in $(\Gamma, \leq)$ together with pairs of integers.

In $\S 4$, the main structure theorem is presented (Theorem 4.8). We prove that every equational theory $E$ of commutative semigroups consists of the identities of some $S$-theory $E(J, m, r)$ and of a set $\pi$, called the remainder of $E$. The remainders are very special sets of finite character: their elements can be always described by listing a finite number of possible forms, and they are, in fact, equivalence relations whose blocks are antichains in the well-quasi-order $(\Gamma, \leq)$. It is the remainders that are responsible for that $\mathscr{L}(\mathrm{Com})$ is not so simple and regular as it could be.

In $\S 5$, we provide an algorithm that gives explicit solutions to the word problem for free semigroups in all varieties of commutative semigroups, and which 
can be even used in small hand computation. We estimate also the computational complexity of the problem by showing that, in general, to decide whether an identity $e$ is a consequence of a finite set of commutative semigroup identities or not is NP-hard.

The lattice $\mathscr{L}(\mathrm{Com})$ is described in $\S 6$. In the description, apart from usual lattice operations on integers, filters, and equivalence relations, also a certain mixed, but rather simple and natural, operation is used.

Throughout the paper, we provide many examples and applications. In the last section, we apply our results to finite semigroups. In particular, we improve Perkins' result [27] on the number of variables in an equational base of a finitely generated commutative semigroup, and characterize those varieties in $\mathscr{L}(\mathrm{Com})$, which are generated by a finite semigroup. The latter turn out to form a sublattice of $\mathscr{L}(\mathrm{Com})$. We show also that various finiteness properties considered for varieties are, in case of varieties of commutative semigroups, equivalent.

An integral part of the study of languages and automata is the study of pseudovarieties of semigroups (classes of finite semigroups closed under the formation of homomorphic images, subsemigroups, and finite direct products; see [29]). Some partial results on pseudovarieties of commutative semigroups were obtained in [2, 3]. In the subsequent article [17], we apply our results to describe all pseudovarieties of commutative semigroups and the lattice they form.

Most of this work was done when the author was visiting the Technische Hochschule in Darmstadt as an Alexander von Humboldt research fellow. It is my pleasure to thank my host, Professor R. Wille, and the participants of his seminar, especially, P. Burmeister, B. Ganter, and Z. Lonc, for interest in the topic of this paper and many helpful remarks. A discussion with M. V. Volkov during the conference in Oberwolfach (July 1991) is gratefully acknowledged. I wish also to thank Professor G. Grätzer for his constructive criticism and continual support.

\section{Preliminaries}

Our approach is equational. Making use of the one-to-one correspondence between varieties and equational theories set up by the Birkhoff theorem, we study equational theories.

Assuming familarity with basic concepts of equational logic and semigroup theory (see, e.g., $[12,39]$ and $[6,20,28]$ ) we start from the following.

1.1. We deal exclusively with equational theories of commutative semigroups; an identity (equation) is written in the form

$$
x_{1}^{\alpha_{1}} \cdots x_{n}^{\alpha_{n}}=x_{1}^{\beta_{1}} \cdots x_{n}^{\beta_{n}}
$$

or occasionally as a $2 \times n$-matrix

$$
\left(\begin{array}{c}
\alpha_{1} \cdots \alpha_{n} \\
\beta_{1} \cdots \beta_{n}
\end{array}\right)
$$

with $\alpha_{i}+\beta_{i}>0$. It is nontrivial if $\alpha_{j} \neq \beta_{j}$ for some $j$. It is regular if $\alpha_{i}, \beta_{i}>0$ for all $i$.

We axiomatize the consequence relation as follows. 
Let $I d$ be the set of matrices (2) with $\alpha_{i}+\beta_{i}>0$. Two matrices that can be obtained from each other by permuting columns or rows are considered equivalent.

A set $E \subseteq I d$ of matrices (identities) is called a closed set (or an equational theory of commutative semigroups, or simply, a theory) if it contains all trivial matrices and the following conditions hold.

For every $e$ of the form (2), if $e$ is in $E$, then the matrices obtained from $e$ by

(E0) forming equivalent matrices,

(E1) adjoining $\left(\begin{array}{l}1 \\ 1\end{array}\right)$ column,

(E2) adjoining $\left(\begin{array}{c}\alpha_{i} \\ \beta_{i}\end{array}\right)$ column, for some $i$,

(E3) replacing $\left(\begin{array}{c}\alpha_{i} \\ \beta_{i}\end{array}\right)$ and $\left(\begin{array}{c}\alpha_{j} \\ \beta_{j}\end{array}\right)$ columns by $\left(\begin{array}{c}\alpha_{i}+\alpha_{j} \\ \beta_{i}+\beta_{j}\end{array}\right)$, for some $i \neq j$, are also in $E$. Moreover, for every $e_{1}, e_{2}$, and $e_{3}$,

$$
e_{1}=\left(\begin{array}{c}
\alpha_{1} \cdots \alpha_{n} \\
\beta_{1} \cdots \beta_{n}
\end{array}\right), \quad e_{2}=\left(\begin{array}{c}
\beta_{1} \cdots \beta_{n} \\
\gamma_{1} \cdots \gamma_{n}
\end{array}\right), \quad e_{3}=\left(\begin{array}{c}
\alpha_{1} \cdots \alpha_{n} \\
\gamma_{1} \cdots \gamma_{n}
\end{array}\right) \text {. }
$$

(E4) If $e_{1}$ and $e_{2}$ are in $E$, then $e_{3}$ is also in $E$.

Rule E1 corresponds to multiplication by a variable. E2 is substitution $x_{i} y_{i}$ for $x_{i}$. E3 is identification of variables $x_{i}$ and $x_{j}$.

The least closed set containing a set of identities $E$ is denoted by $E^{*}$. The completeness theorem in the following form holds: an identity $e \in E^{*}$ if and only if every commutative semigroup that is a model of $E$ also satisfies $e$.

Closed sets ordered by inclusion form a complete lattice with meet $E_{1} \wedge E_{2}=$ $E_{1} \cap E_{2}$, and join $E_{1} \vee E_{2}=\left(E_{1} \cup E_{2}\right)^{*}$. This lattice, which is dually isomorphic to the lattice of varieties of commutative semigroups, will be denoted by $\mathscr{L}_{\text {Com }}$.

The following well-known result is due to Perkins [27].

1.2. Proposition. Every closed set $E \subseteq I d$ is finitely generated. Consequently, $\mathscr{L}_{\text {Com }}$ is countable and has no infinite ascending chains.

1.3. The following notations and conventions are used throughout the paper without further mention.

The letter $e$ denotes always an identity of the form (1). For simplicity, we write sometimes $e=\left(\alpha_{1} \cdots \alpha_{n}, \beta_{1} \cdots \beta_{n}\right)$ or, if we wish to refer to the corresponding sequences, $e=(\mathbf{a}, \mathbf{b})$, where $\mathbf{a}=\left(\alpha_{1}, \ldots, \alpha_{n}\right)$ and $\mathbf{b}=\left(\beta_{1}, \ldots, \beta_{n}\right)$.

Identities of the form $t x=x$ and $t x=t$, where $t$ is a term and $x$ a variable not occurring in $t$, we write briefly $t=1$ and $t=0$, respectively.

By $E_{\varnothing}=\varnothing^{*}$ we denote the theory of all commutative semigroups, i.e., the least element in $\mathscr{L}_{\text {Com }}$. For maximum, minimum, the greatest common divisor, and the least common multiple of two or more integers we write max, min, gcd, and $1 \mathrm{~cm}$, respectively. The notion retract is used in an algebraic sense, i.e., a sublattice $\mathscr{L}_{0}$ of the lattice $\mathscr{L}=(L, \vee, \wedge)$ is called a retract if there is a $(\vee, \wedge)$-epimorphism $\phi$ of $\mathscr{L}$ onto $\mathscr{L}_{0}$ fixing all the points of $\mathscr{L}_{0}$.

1.4. We shall need also the following fundamentals of the theory of quasiorderings.

A relation $\leq$ on a set $\Gamma$ is called a quasi-order if it is reflexive and transitive. Elements $a, b$ satisfying $a \leq b \leq a$ are said to be equivalent. Identifying equivalent elements yields a partial order associated with $\leq$, which often helps 
to visualize the structure of the original quasi-order. An antichain in a quasiordered set is a subset of pairwise noncomparable elements. A chain is a subset in which every two elements are comparable, but no two distinct elements are equivalent. An element $b \in \Gamma$ is minimal if $a \leq b$ implies $b \leq a$.

An order filter, or simply, a filter in a quasi-ordered set $(\Gamma, \leq)$ is a set $J$ with the property $a \in J$ and $a \leq b$ implies $b \in J$ (the name terminal segment is also in use). The set of all filters in the quasi-ordered set $\Gamma$ forms a complete sublattice of the power set of $\Gamma$ (whence, this lattice is, in particular, distributive).

The following result is well known and easy to verify.

1.5. Proposition. For a quasi-ordered set $\Gamma$ the following are equivalent.

(i) Every nonempty subset of $\Gamma$ has at least one and only finitely many nonequivalent minimal elements.

(ii) $\Gamma$ has no infinite antichains or descending chains.

(iii) Every filter $J$ in $\Gamma$ is finitely generated (i.e., there is a finite set $F$ such that $J=\{a: b \leq a$, for some $b \in F\})$.

A quasi-ordered set $\Gamma$ satisfying the equivalent conditions of Proposition 1.5 is said to be well-quasi-ordered. The filters in a well-quasi-ordered set are especially easy to handle because of condition (iii). This condition means actually that every filter is generated and determined uniquely by its minimal elements.

It is also not difficult to observe that the property of being well-quasi-ordered is preserved under many operations, such as taking subsets, extending order, etc. In the sequel, we refer only to taking subsets and extending order, and in addition, to the following result due to Higman [15].

1.6. Proposition. Let $(Q, \leq)$ be a well-quasi-ordered set, and for finite sequences $\mathbf{a}$ and $\mathbf{b}$ of elements of $Q$ define $\mathbf{a} \leq_{m} \mathbf{b}$ if some subsequence of $\mathbf{b}$ majorizes a term by term. Then, the space $Q^{<\omega}$ of all finite sequences over $Q$ is itself well-quasi-ordered.

For other transfer properties and more details on quasi-orderings consult [19] and the references given therein.

1.7. There are many special symbols introduced throughout the paper. For quick reference we list those used many times (and not mentioned in this section), with short explanation followed by the subsection number of the first appearance.

$$
\begin{array}{ll}
\equiv_{N} & \text { a partition of } \mathscr{L}_{\text {Com }} \text { corresponding to the sublattice } \mathscr{L}_{N}, 2.2 . \\
\mathscr{I}_{N}(k, m, r) & \begin{array}{l}
\text { an interval of } \mathscr{L}_{\text {Com }} \text { in the partition } \equiv_{N}, 2.2 . \\
\text { N1-N4 }
\end{array} \\
E(k, m, r) & \text { the conditions in Lemma } 2.3 . \\
\text { the least theory in } \mathscr{I}_{N}(k, m, r), 2.7 \text { and Lemma 2.8. } & \text { the sublattice of theories } E(k, m, r), 2.7 . \\
\mathscr{L}_{N} & \text { the greatest theory in } \mathscr{I}_{N}(k, m, r), 2.13 . \\
\left.\mathscr{L}_{S}, m, r\right) & \text { the sublattice of } S \text {-theories, 3.1. } \\
\Gamma, \Gamma_{+} & \text {the set of finite sequences of nonnegative (resp. positive) } \\
& \text { integers, 3.4. }
\end{array}
$$




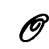

$\mathbf{a} \leq \mathbf{b}$

$\Gamma(e)$

$\Gamma(E)$

$\left[\mathbf{a}_{1}, \ldots, \mathbf{a}_{n}\right]$

$[(m)]$

$k(J), m(J)$

$E(J, m, r)$

$B(J, m, r)$

$d(J)$

$\equiv_{S}$
$\mathscr{I}_{S}(J, m, r)$
$\pi 0-\pi 4$
$E(J, m, r, \pi)$
$\mathscr{L}(J, m, r)$
$\theta, \rho$
$G(J, m, r)$
$E 3^{+}, E 1^{\times}$
$C(J, \pi)$
$E(\mathbf{S}), E(\mathscr{V})$
$k(\mathbf{S}), \ldots, r(\mathscr{V})$
$I d_{n}(\mathbf{S})$

the set of operations on the set $\Gamma$ generated by the operations $g_{i j}$ and $h, 3.4$.

the well-quasi-ordering on the set $\Gamma$ defined in $\S 3.5$.

the sequence associated with an $S$-identity $e, 3.8$.

the set of sequences associated with $S$-identities in $E, 3.8$.

the filter in $(\Gamma, \leq)$ generated by the sequences

$\mathbf{a}_{1}, \ldots, \mathbf{a}_{n}, 3.10$.

the filter generated by the one-element sequence $(m), 3.10$.

integers associated with a filter $J$ in $(\Gamma, \leq), 3.11$.

a theory defined in $\S 3.11$; every $S$-theory is of this form, see Theorem 3.12.

the normal base for $E(J, m, r), 3.14$.

an integer associated with the fundamental antichain of the filter $J, 3.14$.

a partition of $\mathscr{L}_{\text {Com }}$ corresponding to the sublattice $\mathscr{L}_{S}, 4.1$.

an interval of $\mathscr{L}_{\text {Com }}$ in the partition $\equiv_{S}, 4.1$.

the conditions characterizing remainders, 4.7.

an arbitrary theory of $\mathscr{L}_{\text {Com }}$, Theorem 4.8 .

the lattice of the remainders isomorphic to $\mathscr{I}_{S}(J, m, r)$, 4.10.

the least and the greatest elements in $\mathscr{L}(J, m, r), 4.12$.

the greatest theory in the interval $\mathscr{I}_{S}(J, m, r), 4.12$.

additional rules of deduction, 5.1.

the union of those blocks in $\pi$, which have a point in common with $J, 6.1$.

the equational theories of the semigroup $\mathbf{S}$ and the variety

$\mathscr{V}$, respectively, 7.1.

parameters given by $E(\mathbf{S})$ and $E(\mathscr{V}), 7.1$.

the identities of $E(\mathbf{S})$ in at most $n$ variables, 7.4.

\section{First PARTITION}

In this section, using the approach of [24], we partition the lattice $\mathscr{L}_{\text {Com }}$ into intervals and show that this partition is actually a congruence relation on $\mathscr{L}_{\text {Com }}$. This, while done in a quite elementary way, gives the first insight into the structure of $\mathscr{L}_{\text {Com }}$, and will be used later for obtaining more advanced results.

2.1. Lemma. If $E$ contains some nontrivial identity $e$, then there exist $k, r>$ 0 such that

$$
x^{k+r}=x^{k}
$$

is a consequence of $E$, and if $x^{k_{1}+r_{1}}=x^{k_{1}}$ (with $k_{1}, r_{1}>0$ ) is another consequence of $E$, then $k \leq k_{1}$ and $r$ divides $r_{1}$.

Proof. First note that some identity of the form (3) must be in $E^{*}$. To see it, it is enough to identify all variables in $e$. This works, unless $\sum \alpha_{i}=\sum \beta_{i}$. In such a case, to get an inequality, we first choose $j$ such that $\alpha_{j} \neq \beta_{j}$ and then substitute $x_{j}^{2}$ for $x_{j}$.

Now, for $k$ minimal such that (3) is in $E^{*}$ for some $r>0$, choose $r$ minimal. We show that such $k$ and $r$ are as claimed. Suppose, to the contrary, 
that

$$
x^{m+s}=x^{m}
$$

is in $E^{*}$ for some $s>0$ not divisible by $r$. By assumption $m \geq k$. We may assume also that $0<s<r$, because we can use (3) to reduce $s$ modulo $r$. Now, from (3) we have

$$
x^{k+\alpha r}=x^{k}
$$

for any $\alpha \geq 0$. Replacing here $\alpha$ by $\alpha-1$, and multiplying it by $x^{r-s}$ we obtain

$$
x^{k+\alpha r-s}=x^{k+(r-s)}
$$

for any $\alpha \geq 1$. In turn, multiplying (4) by $x^{k+\alpha r-m-s}$ we get

$$
x^{k+\alpha r}=x^{k+\alpha r-s}
$$

$(k+\alpha r \geq m+s)$. Comparing (5), (6), and (7), yields

$$
x^{k+(r-s)}=x^{k}
$$

which contradicts the minimality of $r$.

2.2. Using Lemma 2.1 , we can partition $\mathscr{L}_{\text {Com }}$ into equivalence classes. Following Nelson [24], we add however one more parameter.

Given $k, r>0$ and $m$, with $k \geq m \geq 0$, by $\mathscr{J}_{N}(k, m, r)$ we denote the class of those theories $E$ in $\mathscr{L}_{\text {Com }}$ for which $k$ and $r$ are minimal positive integers given by Lemma 2.1 , and $m$ is the least nonnegative integer such that

$$
x^{m+r} y^{k}=x^{m} y^{k}
$$

is in $E$. Clearly, for $m \geq k,(8)$ is a consequence of (3), and that is why $m \leq k$.

The equivalence relation on $\mathscr{L}_{\text {Com }}$ given by the definition above will be denoted by $\equiv_{N}$ : two theories are $N$-equivalent if they belong to the same class $\mathscr{I}_{N}(k, m, r)$ (note, that under this definition an additional $N$-equivalence class is $\left\{E_{\varnothing}\right\}$ consisting of the theory $E_{\varnothing}$ alone).

2.3. Lemma. Let $E \in \mathscr{I}_{N}(k, m, r)$. If $e \in E$, then the following conditions hold.

(N1) If $\sum \alpha_{i} \neq \sum \beta_{i}$, then both $\sum \alpha_{i}, \sum \beta_{i} \geq k$.

(N2) If $\sum \alpha_{i}=\sum \beta_{i}$, then for every $j$ such that $\alpha_{j} \neq \beta_{j}$ both $\left(\alpha_{j}+\sum \alpha_{i}\right)$, $\left(\beta_{j}+\sum \beta_{i}\right) \geq k$.

(N3) For every $i, \alpha_{i} \equiv \beta_{i}(\bmod r)$.

(N4) For every $i$, if $\alpha_{i} \neq \beta_{i}$, then both $\alpha_{i}, \beta_{i} \geq m$.

Proof. In view of Lemma 2.1 and the argument used in the first part of the proof of that lemma, N1 and N2 are obvious.

For N3, note that in view of Lemma 2.1,r divides $\left(\sum \alpha_{i}-\sum \beta_{i}\right)$. In turn, if we substitute $x_{i}^{2}$ for $x_{i}$, we get that $r$ divides also $\left(\left(\sum \alpha_{i}-\sum \beta_{i}\right)+\left(\alpha_{i}-\beta_{i}\right)\right)$. Hence, $r$ divides $\left(\alpha_{i}-\beta_{i}\right)$, as required.

To prove N4, suppose that $\alpha_{j}<\beta_{j}$ and $\alpha_{j}<m$. Then, identifying all variables in $e$ except $x_{j}$, we get

$$
x^{\alpha_{j}} y^{\alpha}=x^{\beta_{j}} y^{\beta}
$$


where, by N3, $\alpha \equiv \beta(\bmod r)$. Multiplying this by $y^{s}$, where $s$ is chosen so that $\alpha+s, \beta+s \geq k$, and both are equal to $k$ modulo $r$, and then using (3), we obtain

$$
x^{\alpha_{j}} y^{k}=x^{\beta_{j}} y^{k} .
$$

We may assume that $\alpha_{j}=m-1$ (multiplying the identity by a suitable factor, if necessary). Then, using (8), $\beta_{j}$ (which is equal to $\alpha_{j}$ modulo $r$ ) may be reduced to $m+r-1$, thus contradicting the minimality of $m$.

The conditions in Lemma 2.3 play a crucial role in this paper and in the sequel will be referred to as conditions N1-N4.

2.4. Lemma. Let $E$ be a nonempty set of nontrivial identities. Define $k_{0}=$ the greatest $k$ such that $\mathrm{N} 1$ and $\mathrm{N} 2$ hold for every $e$ in $E$. $m_{0}=$ the greatest $m$ such that $\mathrm{N} 4$ holds for every $e$ in $E$.

$r_{0}=$ the greatest $r$ such that $\mathrm{N} 3$ holds for every $e$ in $E$ (= the greatest common divisor of all $\left.\alpha_{i}-\beta_{i}\right)$.

Then, $E^{*} \in \mathscr{I}_{N}\left(k_{0}, m_{0}, r_{0}\right)$.

Proof. Choose $k, m, r$ so that $E^{*} \in \mathscr{J}_{N}(k, m, r)$.

By Lemma 2.3, $k \leq k_{0}, m \leq m_{0}$, and $r$ divides $r_{0}$.

On the other hand, $k_{0}, m_{0}$, and $r_{0}$ as operators on sets of identities are invariant under adjoining consequences to the set (routine checking the rules E0-E4 is left to the reader). In particular, since (3) and (8) are in $E^{*}, k \geq k_{0}$, $m \geq m_{0}$, and $r_{0}$ divides $r$.

Remark. The numbers $k_{0}, m_{0}$, and $r_{0}$ in the lemma above are denoted in [24] by $U(E), V(E)$, and $D(E)$, respectively.

Note also that Lemma 2.4 shows, in particular, that all the classes $\mathscr{I}_{N}(k, m, r)$ are nonempty.

2.5. Lemma. If $E_{1} \in \mathscr{I}_{N}\left(k_{1}, m_{1}, r_{1}\right)$ and $E_{2} \in \mathscr{I}_{N}\left(k_{2}, m_{2}, r_{2}\right)$, then

$$
E_{1} \cap E_{2} \in \mathscr{I}_{N}\left(k_{3}, m_{3}, r_{3}\right),
$$

where $k_{3}=\max \left(k_{1}, k_{2}\right), m_{3}=\max \left(m_{1}, m_{2}\right)$, and $r_{3}=\operatorname{lcm}\left(r_{1}, r_{2}\right)$, and

$$
E_{1} \vee E_{2} \in \mathscr{I}_{N}\left(k_{4}, m_{4}, r_{4}\right) \text {, }
$$

where $k_{4}=\min \left(k_{1}, k_{2}\right), m_{4}=\min \left(m_{1}, m_{2}\right)$, and $r_{4}=\operatorname{gcd}\left(r_{1}, r_{2}\right)$.

The above formulas remain valid for infinite joins, and for infinite meets, as well, provided both corresponding maxima and the least common multiple exist; if not, then the meet is equal to $E_{\varnothing}$.

Proof. Note that as a consequence of (3) and (8) for $k=k_{1}, m=m_{1}$, and $r=r_{1}$ (or, respectively, $k_{2}, m_{2}$, and $r_{2}$ ) we can get (3) and (8) for $k=k_{3}$, $m=m_{3}$, and $r=r_{3}$. Hence, these are in $E_{1} \cap E_{2}$. Combining this with Lemma 2.4 yields the first statement. The statement for $E_{1} \vee E_{2}$ is a direct consequence of Lemma 2.4. The arguments hold in the infinite case as well.

As the main corollary of Lemma 2.5 we have the following.

2.6. Proposition. The relation $\equiv_{N}$ is a congruence relation on $\mathscr{L}_{\text {Com }}$, and every equivalence class $\mathscr{I}_{N}(k, m, r)$ is an interval of $\mathscr{L}_{\text {Com }}$.

Proof. The first statement is immediate from Lemma 2.5. For the second it is enough to observe that if $E_{1} \subseteq E \subseteq E_{2}$ and $E_{1}, E_{2} \in \mathscr{I}_{N}(k, m, r)$ then $E_{1} \cap E, E \vee E_{2} \in \mathscr{I}_{N}(k, m, r)$, and use Lemma 2.5. 
2.7. Following Nelson [24], for $k \geq m$, let $E(k, m, r)$ be the theory generated by (3) and (8) (in [24] it is denoted by $\Omega_{m, k, r}^{*}$ ). By Lemma 2.4, $E(k, m, r) \in$ $\mathscr{I}_{N}(k, m, r)$, and it is actually the least element in the interval $\mathscr{I}_{N}(k, m, r)$. Thus, the set $\mathscr{L}_{N}$ consisting of all the theories $E(k, m, r)$ with $E_{\varnothing}$ adjoined is a set of representatives for $\equiv_{N}$. In order to see that it is also a sublattice of $\mathscr{L}_{\text {Com }}$ we need the following explicit solution for word problems for the corresponding free semigroups.

2.8. Lemma. A nontrivial $e$ belongs to $E(k, m, r)$ if and only if there are $i$ and $j$ (not necessarily distinct) such that $\alpha_{i}, \beta_{j} \geq k$ and conditions $\mathrm{N} 3$ and N4 are satisfied.

Proof. First, observe that the existence of $j$ with $\alpha_{j} \geq k$ is invariant under the rules E0-E4. This and Lemma 2.3(N3-N4) yield the "only if" part.

For the "if" part, let $e$ satisfy the conditions given in the lemma. Then, using (8) we get

$$
x_{1}^{\alpha_{1}} \cdots x_{n}^{\alpha_{n}}=x_{1}^{\gamma_{1}} \cdots x_{n}^{\gamma_{n}},
$$

where $\gamma_{i} \geq k$, whenever $\alpha_{i} \geq m$, and $\gamma_{i}=\alpha_{i}$, otherwise. Moreover, $\gamma_{i} \equiv \alpha_{i}$ $(\bmod r)$. The same argument holds for $x_{1}^{\beta_{1}} \cdots x_{n}^{\beta_{n}}$. Hence, using (3) completes the proof.

The lemma above appears in [24] as Theorem 2.1. The next lemma together with the fact that $E(k, m, r) \in \mathscr{I}_{N}(k, m, r)$ covers Theorems 1.1, 2.2, 2.3, and remarks in $\S 2.1$ of [24]. We state it as a lemma here, because we are going to obtain a much more general result (Theorem 6.2).

2.9. Lemma. Let $E_{1}=E\left(k_{1}, m_{1}, r_{1}\right)$ and $E_{2}=E\left(k_{2}, m_{2}, r_{2}\right)$. Then the following hold.

(i) $E_{1} \supseteq E_{2}$ if and only if $k_{1} \leq k_{2}, m_{1} \leq m_{2}$, and $r_{1}$ divides $r_{2}$.

(ii) $E_{1} \cap E_{2}=E\left(k_{3}, m_{3}, r_{3}\right)$, where $k_{3}=\max \left(k_{1}, k_{2}\right), m_{3}=\max \left(m_{1}, m_{2}\right)$, and $r_{3}=\operatorname{lcm}\left(r_{1}, r_{2}\right)$.

(iii) $E_{1} \vee E_{2}=E\left(k_{4}, m_{4}, r_{4}\right)$, where $k_{4}=\min \left(k_{1}, k_{2}\right), m_{4}=\min \left(m_{1}, m_{2}\right)$, and $r_{4}=\operatorname{gcd}\left(r_{1}, r_{2}\right)$.

As in Lemma 2.5, the above formulas remain valid for infinite joins and meets. Thus $\mathscr{L}_{N}$ is a complete sublattice of $\mathscr{L}_{\text {Com }}$.

Proof. (i) and (ii) are easy consequences of Lemma 2.8. For (iii), we infer: by Lemma $2.5, E_{1} \vee E_{2} \in \mathscr{I}_{N}\left(k_{4}, m_{4}, r_{4}\right)$, by (i), $E_{1}, E_{2} \subseteq E\left(k_{4}, m_{4}, r_{4}\right)$, and in consequence, $E_{1} \vee E_{2} \subseteq E\left(k_{4}, m_{4}, r_{4}\right)$. Since $E\left(k_{4}, m_{4}, r_{4}\right)$ is the least element in $\mathscr{I}_{N}\left(k_{4}, m_{4}, r_{4}\right)$, the result follows.

Now, combining Lemmas 2.9 and 2.5 , we get a result showing that $\mathscr{L}_{N}$, in fact, determines to some extent "the shape" of $\mathscr{L}_{\text {Com }}$.

2.10. Proposition. For every $E \in \mathscr{L}_{\mathrm{Com}}$ define $\varphi(E)$ as the greatest element in $\mathscr{L}_{N}$ contained in $E$. Then $\varphi$ is a homomorphism of $\mathscr{L}_{\text {Com }}$ onto $\mathscr{L}_{N}$. In particular, $\mathscr{L}_{N}$ is a retract of $\mathscr{L}_{\text {Com }}$, and $\mathscr{L}_{N} \cong \mathscr{L}_{\mathrm{Com}} / \equiv_{N}$.

2.11. Lemma 2.8 can be used also to determine the cardinality of the $n$ generated free semigroups $\mathbf{F}_{n}(E(k, m, r))$ in the varieties corresponding to theories $E(k, m, r)$. We can distinguish two types of words $x_{1}^{\alpha_{1}} \cdots x_{n}^{\alpha_{n}}$ in such semigroups: those with all $\alpha_{i}<k$ (there are $k^{n}-1$ of them), and those 
with some $\alpha_{i} \geq k$. In the latter case all exponents except one can be reduced modulo $r$ to be less than $m+r$, and it is not hard to see that there are precisely $(m+r)^{n}-m^{n}$ distinct words. Hence we have

2.12. Corollary. The cardinality $\left|\mathbf{F}_{n}(E(k, m, r))\right|=k^{n}+(m+r)^{n}-m^{n}-1$ for all $n \geq 0$.

It is also not difficult to describe the greatest elements in each $\mathscr{I}_{N}(k, m, r)$. Let us denote it by $G(k, m, r)$. Then, by Lemmas 2.3 and 2.4, we have

2.13. Corollary. For a nontrivial $e, e \in G(k, m, r)$, if and only if conditions N1-N4 are satisfied.

Using this we prove

2.14. Proposition. Given $k, r>0$ and $k \geq m \geq 0$ let $E$ consist of the following identities

$$
y^{m} x_{1} \cdots x_{k-m}=y^{m+r} x_{1} \cdots x_{k-m}
$$

and, in addition, for every $u \geq m$ and such that $2 u+r<k$

$$
z^{u} y^{u+r} x_{1} \cdots x_{q}=z^{u+r} y^{u} x_{1} \cdots x_{q},
$$

where $q=\max (0, k-3 u-r)$.

Then, $E^{*}=G(k, m, r)$.

Proof. $E^{*} \in \mathscr{I}_{N}(k, m, r)$ by Lemma 2.4. In view of Corollary 2.13 we need only show that every nontrivial $e$ satisfying N1-N4 belongs to $E^{*}$. According to N1-N2 there are two possibilities for $e$.

1. If $\sum \alpha_{i}, \sum \beta_{i} \geq k$, then (9) applies and the claim is obvious.

2. If $\sum \alpha_{i}=\sum \beta_{i}<k$, then choose $i$ such that $\alpha_{i} \neq \beta_{i}$. If, for instance, $\alpha_{i}<\beta_{i}$, then in this case, there is also $j$ such that $\alpha_{j}>\beta_{j}$. Let $u=\min \left(\alpha_{i}, \beta_{j}\right)$. We wish to show first that now (10) can be applied.

Assume, for instance, that $u=\alpha_{i}$. Then, since $\alpha_{j} \equiv \beta_{j}(\bmod r)$ and $\alpha_{j}>$ $\beta_{j}, \alpha_{j} \geq u+r$. It follows that $2 u+r \leq \sum \alpha_{i}<k$. Also, by virtue of N4, $u \geq m$. It remains to show that $\sum \alpha_{i} \geq 2 u+r+q$. To this end it is enough to observe that the latter is equivalent to $\sum \alpha_{i} \geq k-u$, which is an immediate consequence of $\mathrm{N} 2$.

Repeated application of (10) proves that also in case 2, $e \in E^{*}$.

2.15. By Corollary $2.13, G\left(k_{1}, m_{1}, r_{1}\right) \cap G\left(k_{2}, m_{2}, r_{2}\right)=G\left(k_{3}, m_{3}, r_{3}\right)$, where $k_{3}, m_{3}$, and $r_{3}$ are as in Lemma 2.5. But $G\left(k_{1}, m,{ }_{1}, r_{1}\right) \vee G\left(k_{2}, m_{2}, r_{2}\right)$ may fail to equal $G\left(k_{4}, m_{4}, r_{4}\right)$. For example, using Proposition 2.14, it is easy to find that $G(2,1,1)=\left\{x^{2}=x^{3}\right\}^{*}, G(3,0,1)=\{x y z=0\}^{*}$, while $G(2,0,1)=\{x y=0\}^{*} \neq\left\{x^{2}=x^{3}, x y z=0\right\}^{*}$ (the latter inequality one may easily prove directly or check using Theorem 3.12; see $\S 3.17$ ).

2.16. To close the section, let us have a look at the content of some intervals $\mathscr{I}_{N}(k, m, r)$. Obviously, $E(1,0,1)=\{x=y\}^{*}$ is the greatest element of $\mathscr{L}_{\text {Com }}$, and therefore $\mathscr{I}_{N}(1,0,1)$ is a class consisting of only $\{x=y\}^{*}$. Further, we have $E(1,0, r)=G(1,0, r)=\left\{x^{r}=1\right\}^{*}$. Hence, for every $r \geq 1$, $\mathscr{I}_{N}(1,0, r)$ consists only of one element: the equational theory of abelian groups of exponent $r$. Those with $r$ prime form an infinite set of coatoms in $\mathscr{L}_{\text {Com }}$ (the remaining two are $E(1,1,1)$ and $G(2,0,1)$ below). 
Also, $E(k, k, r)=G(k, k, r)=\left\{x^{k+r}=x^{k}\right\}^{*}$, and the classes $\mathscr{I}_{N}(k, k, r)$ are singletons. In particular, $E(k, k, 1)$ are theories of aperiodic semigroups, and $E(1,1,1)$ is the theory of semilattices.

$E(k, 0,1)=\left\{x^{k}=0\right\}^{*}$ are theories of nil-semigroups. By Proposition 2.14, $G(k, 0,1)=\left\{x_{1} \cdots x_{k}=0, \ldots\right\}^{*}$, where dots mean the possibility of some further identities in accordance with the proposition. (In particular, $G(2,0,1)=\{x y=0\}^{*}, G(3,0,1)=\{x y z=0\}^{*}$, but $G(4,0,1)=$ $\left.\left\{x y z t=0, x y^{2}=x^{2} y\right\}^{*}\right)$. Hence, $\mathscr{J}_{N}(k, 0,1)$ consists of more than one element, and as a matter of fact, every such interval is infinite. For example, $\mathscr{J}_{N}(2,0,1)$ consists of the chain of the theories $\left\{x^{2}=0, x_{1} \cdots x_{k}=0\right\}^{*}$.

Finally, $E(2,0, r)=\left\{x^{r} y^{2}=y^{2}\right\}^{*}, G(2,0, r)=\left\{x^{r} y z=y z\right\}^{*}$, and $\mathscr{I}_{N}(2,0, r)$ are again infinite. We shall see later $(\S 3.17)$ that $\mathscr{I}_{N}(k, m, r)$ always either consists of one element, whenever $k=m$ or $k=1$, or is infinite, otherwise.

Note also that, in view of Lemma $2.5, \mathscr{L}_{\text {Com }}$ has no atoms, and the least element $E_{\varnothing}$ is meet irreducible.

\section{SChWABAUER SUblatTice}

In order to obtain a finer partition, we would like to apply the successful approach of $\S 2$ to the sublattice $\mathscr{L}_{S}$ of the Schwabauer theories [36]. The problem is however that no description of $\mathscr{L}_{S}$ is known. The aim of this section is to provide such a one.

3.1. Recall that a nontrivial identity $e$ of the form (1) is called an $S$-identity if $\alpha_{i} \leq \beta_{i}$ for all $i$. A theory generated by one or more $S$-identities is called an $S$-theory. The subset $\mathscr{L}_{S}$ of $\mathscr{L}_{\text {Com }}$ consisting of all $S$-theories and the theory $E_{\varnothing}$ is a sublattice of $\mathscr{L}_{\text {Com }}$ [36]. Since every $E(k, m, r)$ is defined by $S$-identities, we have $\mathscr{L}_{N} \subseteq \mathscr{L}_{S}$.

Our description is based on the following.

3.2. Lemma. Let $E \in \mathscr{I}_{N}(k, m, r)$, and let

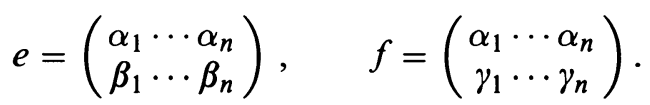

If $e$ is an $S$-identity with $\alpha_{i} \leq \beta_{i}$, then the following are equivalent.

(i) $e \in E$.

(ii) Some $f$ with $\gamma_{i}=\alpha_{i}$ whenever $\alpha_{i}<m$, and $\gamma_{i} \geq k, \gamma_{i} \equiv \alpha_{i}(\bmod r)$, otherwise, is in $E$.

(iii) All $f$ with $\gamma_{i}=\alpha_{i}+k_{i} r$, for all $k_{1}, \ldots, k_{n} \geq 0$, such that $k_{i}=0$ whenever $\alpha_{i}<m$, are in $E$.

(iv) For some $j$ with $\alpha_{j} \geq m, f$ with $\gamma_{j}=\alpha_{j}+r$, and $\gamma_{i}=\alpha_{i}$, otherwise, is in $E$.

Proof. First we show that (i)-(iii) are equivalent.

(i) $\Rightarrow$ (ii). Suppose that $\beta_{i}=\alpha_{i}+k_{i} r$. Then, multiplying $e$ by $x_{i}^{k_{i} r}$ for all $i$, and comparing the resulting identity with $e$ itself, we deduce that $f \in E$ with $\gamma_{i}=\alpha_{i}+2 k_{i} r$. Repeating this several times and using (3), we obtain (ii).

(ii) $\Rightarrow$ (iii). It is enough to multiply $f$ in (ii) by $x_{i}^{k_{i} r}$ for all $i$, and again compare the resulting identity with $f$ itself, applying (3).

(iii) $\Rightarrow$ (i) . Trivial. 
Since also (iii) $\Rightarrow$ (iv) trivially holds, to complete the proof, it is enough to show, e.g., (iv) $\Rightarrow$ (ii).

(iv) $\Rightarrow$ (ii). Let $j$ be fixed. Multiplying $f$ in (iv) by $x_{j}^{r}$, and using an argument analogous to that in (i) $\Rightarrow$ (ii), we obtain that $f$ with $\gamma_{j} \geq k$, $\gamma_{j} \equiv \alpha_{j}(\bmod r)$, and $\gamma_{i}=\alpha_{i}$, otherwise, is in $E$. Now, (ii) follows easily from (8).

3.3. The lemma above shows that every sequence $\left(\alpha_{1}, \ldots, \alpha_{n}\right)$ gives rise to a class of $S$-identities that are all equivalent in $\mathscr{I}_{N}(k, m, r)$. Moreover, in view of (iv), if $m=0$, then $\alpha_{i}=0$ play no role and can be omitted. Also the arrangement of elements plays no role. This suggests a possibility of expressing the consequence relation in terms of a relation on finite sequences (rather than matrices), and we proceed to describe a relevant order.

3.4. Let $\Gamma$ be the set of finite sequences $\left(\alpha_{1}, \ldots, \alpha_{n}\right)$ of nonnegative integers such that at least one $\alpha_{i} \neq 0$. The following set $\mathscr{O}$ of operations on the set $\Gamma$ plays a crucial role in the sequel. Given a sequence $\left(\alpha_{1}, \ldots, \alpha_{n}\right) \in \Gamma$, we define unary operations $g_{i j}$ and $h$ on $\Gamma$ by

$$
g_{i j}\left(\left(\alpha_{1}, \ldots, \alpha_{n}\right)\right)=\left(\alpha_{1}, \ldots, \alpha_{i}+\alpha_{j}, \ldots, \alpha_{n}\right)
$$

(where $\left(\alpha_{1}, \ldots, \alpha_{i}+\alpha_{j}, \ldots, \alpha_{n}\right)$ is a sequence obtained from $\left(\alpha_{1}, \ldots, \alpha_{n}\right)$ by replacing $\alpha_{i}$ by $\alpha_{i}+\alpha_{j}$, and deleting $\alpha_{j}$, provided $i, j \leq n$ and $i \neq j$; otherwise, $g_{i j}$ is the identity operation on $\Gamma$ ), and

$$
h\left(\left(\alpha_{1}, \ldots, \alpha_{n}\right)\right)=\left(\alpha_{1}, \ldots, \alpha_{n}, 1\right) .
$$

By $\mathscr{O}$ we denote the least composition closed set of operations on $\Gamma$ containing all $g_{i j}$, and $h$. Note that, in particular, the identity operation $g_{i i}$, also called trivial, is in $\mathscr{O}$.

3.5. Now, a quasi-order relation $\leq$ on $\Gamma$ is defined as follows.

$$
\left(\alpha_{1}, \ldots, \alpha_{n}\right) \leq\left(\beta_{1}, \ldots, \beta_{m}\right)
$$

if there exists $f \in \mathscr{O}$ such that $f\left(\left(\alpha_{1}, \ldots, \alpha_{n}\right)\right)$ and $\left(\beta_{1}, \ldots, \beta_{m}\right)$ differ at most in the arrangement of elements and the number of zeros occurring in the sequence. (In other words, $\left(\beta_{1}, \ldots, \beta_{m}\right)$ can be obtained from $\left(\alpha_{1}, \ldots, \alpha_{n}\right)$ by applying operations $g_{i j}, h$, permutations, deleting, and adjoining zeros.) Reflexivity and transitivity of $\leq$ are immediate. It is also easy to see that two sequences are equivalent if and only if they differ at most in the arrangement of elements and the number of zeros. The relation has the following natural combinatorial meaning.

3.6. Lemma. $\left(\alpha_{1}, \ldots, \alpha_{n}\right) \leq\left(\beta_{1}, \ldots, \beta_{m}\right)$ if and only if there is a partition $\pi$ of the set $\{1, \ldots, n\}$ and a one-to-one mapping $\psi$ from the set $B_{\pi}$ of the blocks of $\pi$ into the set $\{1, \ldots, m\}$ such that for every $B \in B_{\pi}$,

Proof. Trivial.

$$
\sum_{i \in B} \alpha_{i} \leq b_{\psi(B)} .
$$

Observing that for $\Gamma=Q^{<\omega}$ the quasi-order $\leq$ extends the order $\leq_{m}$ defined in Proposition 1.6, we have also the following. 
3.7. Lemma. The relation $\leq$ is a well-quasi-ordering on $\Gamma$.

3.8. Now, for every $S$-identity $e$ with $\alpha_{i} \leq \beta_{i}$, we define $\Gamma(e)=\left(\alpha_{1}, \ldots, \alpha_{n}\right) \in$ $\Gamma$, and for a set of identities $E$ by $\Gamma(E)$ we denote the subset of $\Gamma$ consisting of all sequences $\Gamma(e)$ corresponding to $S$-identities $e$ in $E$. Then, the connection between $S$-theories and filters in the quasi-order $\leq$ on $\Gamma$ is the following.

3.9. Lemma. Let $S$ be a set of $S$-identities, and suppose that $S^{*} \in \mathscr{I}_{N}(k, m, r)$. Then, a nontrivial $e \in S^{*}$ if and only if $e$ satisfies conditions N1-N4 and both $\left(\alpha_{1}, \ldots, \alpha_{n}\right)$ and $\left(\beta_{1}, \ldots, \beta_{n}\right)$ belong to the filter $J$ in $(\Gamma, \leq)$ generated by $\Gamma(S)$. In particular, $\Gamma\left(S^{*}\right)=J$.

Proof. First note, that if some $\mathbf{b}=\left(\beta_{1}, \ldots, \beta_{m}\right)$ belongs to the filter generated by $\Gamma(S)$, then an $S$-identity $e$ with $\Gamma(e)=\mathbf{b}$ is in $S^{*}$. Indeed, if $\mathbf{a}=\left(\alpha_{1}, \ldots, \alpha_{n}\right) \in \Gamma(S)$ is such that $f\left(\left(\alpha_{1}, \ldots, \alpha_{n}\right)\right)$ is equivalent to $\left(\beta_{1}, \ldots, \beta_{m}\right)$ for some $f \in \mathcal{O}$ (i.e., $\left.\mathbf{a} \leq \mathbf{b}\right)$, and $e_{1} \in S$ such that $\Gamma\left(e_{1}\right)=\mathbf{a}$, then a desired identity can be obtained from $e_{1}$ by applying rules E3, E1, and E0 in accordance with how $f$ is composed from $g_{i j}$ and $h$, respectively.

Whence, to prove the "if" part of the lemma it is enough to compare the $S$ identities corresponding to $\left(\alpha_{1}, \ldots, \alpha_{n}\right)$ and $\left(\beta_{1}, \ldots, \beta_{n}\right)$ given in the form of Lemma 3.2(ii), applying Lemma 2.3 and E4.

For the "only if" part we induct on the length of the proof of $e$ from $\Gamma(S)$. If $e \in \Gamma(S)$, then the statement is obviously true. It remains to check that if $e$ (and $f$ ) has (have) the property in question, then so does every identity obtained by applying the rules E0-E4. This is routine and is left to the reader.

$\Gamma\left(S^{*}\right)=J$ by virtue of the first statement of the proof.

3.10. It follows from Lemma 3.9 that every $S$-theory $E$ can be generated by a set of $S$-identities corresponding to a filter $J$; namely, $J=\Gamma(E)$. In order to establish a one-to-one correspondence between $S$-theories and filters, we first make some observations. In the sequel, by $\left[\mathbf{a}_{1}, \ldots, \mathbf{a}_{n}\right]$ we denote the filter generated by the sequences $\mathbf{a}_{1}, \ldots, \mathbf{a}_{n}$. In particular, $[(m)]$ denotes the (principal) filter generated by the one-element sequence $(m)$. For $m=0$, we define $[(0)]=[(1)]=\Gamma$.

3.11. If $E \in \mathscr{I}_{N}(k, m, r)$, then the one-element sequence $(k)$ belongs to $J$, and $k$ is the least with this property. Given $J$ let us denote the least such integer by $k(J)$. Moreover, if $\left(\alpha_{1}, \ldots, \alpha_{n}\right) \in J$, then in view of Lemma 2.3, and since $S$-identities are assumed to be nontrivial, the inequality $\max \left(\alpha_{1}, \ldots, \alpha_{n}\right)$ $\geq m$ is satisfied. The least integer among $\max \left(\alpha_{1}, \ldots, \alpha_{n}\right)$, for all $\left(\alpha_{1}, \ldots, \alpha_{n}\right)$ $\in J$, we denote by $m(J)$.

Now, given $m, r$, and a filter $J$ such that $m(J) \geq m$, let $S$ be a set of $S$ identities corresponding to $J$ and satisfying conditions N1-N4, with $k=k(J)$, and let us denote $S^{*}$ by $E(J, m, r)$. Then, since $(k) \leq(k, m) \in J$, we have $S^{*}=E(J, m, r) \in \mathscr{I}_{N}(k, m, r)$, and in consequence the hypothesis of Lemma 3.9 holds.

Note, that for $\left(\alpha_{1}, \ldots, \alpha_{n}\right),\left(\beta_{1}, \ldots, \beta_{n}\right) \in J$ conditions N1-N2 for $k=$ $k(J)$ are always satisfied, whence they can be omitted. On the other hand, $m(J) \geq m$ means that $J \subseteq[(m)]$.

Note also, that elements of $J$ represent distinct $S$-identities, and it follows, by Lemma 3.9 , that $E\left(J_{1}, m_{1}, r_{1}\right) \supseteq E\left(J_{2}, m_{2}, r_{2}\right)$ implies, in particular, $J_{1} \supseteq$ $J_{2}$. 
Combining all these remarks with Lemma 2.5, we obtain the following description of the lattice $\mathscr{L}_{S}$.

3.12. Theorem. A theory $E \in \mathscr{L}_{\text {Com }}$ is an $S$-theory iff $E=E(J, m, r)$, for some $m \geq 0, r>0$, and a nonempty order filter $J \subseteq[(m)]$. Then, a nontrivial $e \in E$ if and only if $e$ satisfies conditions N3-N4 and both $\left(\alpha_{1}, \ldots, \alpha_{n}\right)$, $\left(\beta_{1}, \ldots, \beta_{n}\right) \in J$. Moreover, if $E_{1}=E\left(J_{1}, m_{1}, r_{1}\right)$ and $E_{2}=E\left(J_{2}, m_{2}, r_{2}\right)$, then the following hold.

(i) $E_{1} \supseteq E_{2}$ if and only if $J_{1} \supseteq J_{2}, m_{1} \leq m_{2}$, and $r_{1}$ divides $r_{2}$.

(ii) $E_{1} \cap E_{2}=E\left(J_{1} \cap J_{2}, \max \left(m_{1}, m_{2}\right), \operatorname{lcm}\left(r_{1}, r_{2}\right)\right)$.

(iii) $E_{1} \vee E_{2}=E\left(J_{1} \cup J_{2}, \min \left(m_{1}, m_{2}\right), \operatorname{gcd}\left(r_{1}, r_{2}\right)\right)$.

These formulas remain valid for infinite joins and meets except that if the intersection of the filters is empty or if the maximum or the common multiple in question does not exist, then the meet equals $E_{\varnothing}$.

Note, that the intersection of two filters $J_{1} \cap J_{2}$ (of a finite number of filters) is never empty, since for $k=\max \left(k\left(J_{1}\right), k\left(J_{2}\right)\right)$, we have $(k) \in J_{1} \cap J_{2}$. Moreover, $E(k, m, r)=E([(k)], m, r)$.

3.13. Corollary. $\mathscr{L}_{S}$ is a distributive sublattice of $\mathscr{L}_{\text {Com }}$ closed under infinite joins and meets, and containing $\mathscr{L}_{N}$.

3.14. We present a finite equational base for $E(J, m, r)$ consisting only of $S$-identities and involving the least possible number of variables. It determines in a straightforward way the filter $J$ and the theory $E(J, m, r)$; therefore, it is called normal.

A maximal antichain of minimal elements in $J$ is called fundamental if it consists only of increasing sequences with no zeros. Clearly, such an antichain exists and is unique and, by remarks in $\S 1.4$, it generates the filter $J$. By $d(J)$ we denote the maximal length of the sequence in the fundamental antichain of $J$, which equals, obviously, the least number such that $J$ can be generated by sequences of the length not exceeding $n$. (We refer to this number in further sections.)

Now, by $B(J, m, r)$ we denote the set of identities

$$
x_{1}^{\alpha_{1}+r} x_{2}^{\alpha_{2}} \cdots x_{n}^{\alpha_{n}}=x_{1}^{\alpha_{1}} \cdots x_{n}^{\alpha_{n}},
$$

for every $\left(\alpha_{1}, \ldots, \alpha_{n}\right)$ in the fundamental antichain of $J$, and the identity (8), in addition, whenever $m$ is not already determined by the identities (11) themselves (i.e., whenever $\alpha_{1}>m$ for all sequences $\left(\alpha_{1}, \ldots, \alpha_{n}\right)$ in the fundamental antichain of $J$ ).

Then, combining Theorem 3.12 with Lemma 3.7 and Proposition 1.5, we have the following.

3.15. Corollary. Every $S$-theory $E(J, m, r)$ is generated by a finite set of $S$ identities; in particular, $B(J, m, r)$ is an equational base for $E(J, m, r)$.

3.16. Theorem 3.12 may be applied, in particular, to theories generated by irregular identities, that is, those which have a variable occurring only on one side. It is not difficult to see that every such identity is equivalent to a pair of $S$-identities. For example, the identity

$$
x^{2} y^{7}=x^{3} y^{4} z^{2}
$$


is equivalent to

$$
x^{2} y^{7}=x^{5} y^{8} z^{2}, \quad x^{3} y^{4} z^{2}=x^{5} y^{8} z^{2}
$$

(just substitute $x y^{2} z$ for $z$ in (12)). In particular, the theory generated by (12) is $E([(2,3,4)], 0,1)$ (note, that $(2,7) \in[(2,3,4)])$. Consequently, the identity $x^{3} y^{6}=x^{4} z^{5}$ is a consequence of (12), while, for example, $x^{3} y^{3} z^{3}=$ $x^{2} y^{7} z^{3}$ is not.

3.17. We check the inequality in $\S 2.15$.

$$
G(2,0,1)=\{x y=0\}^{*}=E([(1,1)], 0,1),
$$

while

$$
\left\{x^{2}=x^{3}, x y z=0\right\}=E([(2),(1,1,1)], 0,1) .
$$

Also the statement in $\S 2.16$ concerning the cardinality of $\mathscr{I}_{N}(k, m, r)$ can be easily verified. Suppose that $k>m$ and $k>1$. Then, for every filter $J_{s}^{k}=[(k),(k-1, \ldots, k-1)]$, where $k-1$ occurs $s \geq 2$ times, we have $k(J)=k$, and the theories $E\left(J_{s}^{k}, m, r\right)$ are pairwise distinct. All of them lie in the interval $\mathscr{I}_{N}(k, m, r)$; whence, it is infinite.

\section{General STRUCTURE THEOREM}

In this section, we use the sublattice $\mathscr{L}_{S}$ to partition $\mathscr{L}_{\text {Com }}$ into smaller intervals. We follow our approach in $\S 2$. Although the corresponding partition is no longer a congruence relation, the intervals are already small enough to yield a general structure theorem for theories $E \in \mathscr{L}_{\text {Com }}$.

4.1. Given $m \geq 0, r>0$, and $\varnothing \neq J \subseteq[(m)]$, let $\mathscr{I}_{S}(J, m, r)$ be the class of all theories $E \in \mathscr{L}_{\text {Com }}$, such that the join of all $S$-theories contained in $E$ is equal to $E(J, m, r)$ (in other words, such that $E(J, m, r)$ is the greatest $S$-theory contained in $E$ ). The corresponding equivalence relation having as blocks the classes $\mathscr{I}_{S}(J, m, r)$ and $\left\{E_{\varnothing}\right\}$ we denote by $\equiv_{S}$.

Since (3) and (8) are $S$-identities, $\mathscr{I}_{S}(J, m, r) \subseteq \mathscr{I}_{N}(k(J), m, r)$. Hence, $\equiv_{S} \subseteq \equiv_{N}$. The least element in $\mathscr{I}_{S}(J, m, r)$ is obviously $E(J, m, r)$. Furthermore, if $E_{1}, E_{2} \in \mathscr{I}_{S}(J, m, r)$ and $E_{1} \subseteq E \subseteq E_{2}$, then all $E, E_{1} \cap E_{2}$, $E_{1} \vee E_{2} \in \mathscr{I}_{S}(J, m, r)$ (cf. Theorem 3.12). This is also true for infinite meets and joins. Thus, we have the following.

4.2. Proposition. Each equivalence class $\mathscr{I}_{S}(J, m, r)$ is an interval of $\mathscr{L}_{\text {Com }}$. Moreover, $\mathscr{I}_{S}(J, m, r) \subseteq \mathscr{I}_{N}(k(J), m, r)$.

In view of Lemma 2.5 , we have also

4.3. Lemma. If $E_{1} \in \mathscr{I}_{S}\left(J_{1}, m_{1}, r_{1}\right)$ and $E_{2} \in \mathscr{I}_{S}\left(J_{2}, m_{2}, r_{2}\right)$, then the following hold.

(i) $E_{1} \cap E_{2} \in \mathscr{I}_{S}\left(J_{1} \cap J_{2}, \max \left(m_{1}, m_{2}\right), \operatorname{lcm}\left(r_{1}, r_{2}\right)\right)$,

(ii) $E_{1} \vee E_{2} \in \mathscr{S}_{S}\left(J, \min \left(m_{1}, m_{2}\right), \operatorname{gcd}\left(r_{1}, r_{2}\right)\right)$, for some $J \supseteq J_{1} \cup J_{2}$.

As in Theorem 3.12, these formulas remain valid for infinite joins and meets.

In $\S 6.1$, we shall see that $J$ in (ii) may be different from $J_{1} \cup J_{2}$, and therefore $\equiv_{S}$ is not a congruence relation. 
4.4. Let $E \in \mathscr{I}_{S}(J, m, r)$ and $e$ be an identity. Our aim is now to find necessary conditions for $e$ to belong to $E$, analogous to those in Lemma 2.3, and such that a counterpart of Corollary 2.13 holds.

Obviously, at first, conditions N1-N4 must be satisfied, since $\mathscr{I}_{S}(J, m, r) \subseteq$ $\mathscr{I}_{N}(k, m, r)$. Is is also not difficult to observe the following.

4.5. Lemma. Let $E \in \mathscr{I}_{S}(J, m, r)$ and let $e$ of the form (1) be in $E$. Then

(i) $\left(\alpha_{1}, \ldots, \alpha_{n}\right) \in J$ if and only if $\left(\beta_{1}, \ldots, \beta_{n}\right) \in J$.

(ii) For every $i$ such that $\alpha_{i} \neq \beta_{i}$, both $\left(\alpha_{1}, \ldots, \alpha_{n}, \alpha_{i}\right)$ and $\left(\alpha_{1}, \ldots, \alpha_{n}\right.$, $\left.\beta_{i}\right) \in J$.

Proof. For (i), suppose that $\left(\alpha_{1}, \ldots, \alpha_{n}\right) \in J$. Then, by Lemma 3.2(iii) an $S$-identity

$$
x_{1}^{\alpha_{1}} \cdots x_{n}^{\alpha_{n}}=x_{1}^{\gamma_{1}} \cdots x_{n}^{\gamma_{n}}
$$

with $\gamma_{i} \geq \beta_{i}$ is in $E$. By E4, the $\alpha$ 's can be replaced by $\beta$ 's. Hence, $\left(\beta_{1}, \ldots, \beta_{n}\right) \in J$, too.

For (ii), note that, by E1, $\left(\alpha_{1} \cdots \alpha_{n} \beta_{i}, \beta_{1} \cdots \beta_{n} \beta_{i}\right) \in E$, and by E2, $\left(\alpha_{1} \cdots\right.$ $\left.\alpha_{n} \alpha_{i}, \beta_{1} \cdots \beta_{n} \beta_{i}\right) \in E$. Hence, by E4, $\left(\alpha_{1} \cdots \alpha_{n} \alpha_{i}, \alpha_{1} \cdots \alpha_{n} \beta_{i}\right) \in E$. The latter is an $S$-identity, and the result follows by the definition of $\mathscr{I}_{S}(J, m, r)$.

We note also the following.

4.6. Lemma. If $e=(\mathbf{a}, \mathbf{b})$ is an $S$-identity with $\mathbf{a}, \mathbf{b} \notin J$, then $e$ has $a$ consequence $e_{1}=\left(\mathbf{a}_{1}, \mathbf{b}_{1}\right)$ such that $\mathbf{a}_{1} \in J$, while $\mathbf{b}_{1} \notin J$.

Proof. Assume that $\beta_{i}=\alpha_{i}+\delta_{i}, \delta_{i} \geq 0$, for all $i$, and $\delta_{j}>0$ for some $j$. Multiplying $e$ by suitable factors, we obtain the $S$-identity $(\mathbf{b}, \mathbf{c})$, with $\mathbf{c}=\left(\beta_{1}+\delta_{1}, \ldots, \beta_{n}+\delta_{n}\right)$. If $\mathbf{c} \in J$, we are done; if not, we repeat the argument. Since every $\left(\gamma_{1}, \ldots, \gamma_{n}\right)$ with $\gamma_{j} \geq k(J)$ for some $j$ is in $J$, after a finite number of steps we get the result.

4.7. Now, suppose that $E \in \mathscr{I}_{S}(J, m, r)$. Then, we can write

$$
E=E(J, m, r) \cup \pi
$$

where $\pi$ is just $E \backslash E(J, m, r)$ with trivial identities of the form (a, a), a $\notin J$, adjoined. We shall call it the remainder of the theory $E$, since, as we shall see, it is a very special and relatively small part of $E$. We proceed to establish some properties of the remainder.

At first, if $(\mathbf{a}, \mathbf{b}) \in E$, then since $\mathscr{I}_{S}(J, m, r) \subseteq \mathscr{I}_{N}(k, m, r)$ for $k=$ $k(J)$, it satisfies conditions $\mathrm{N} 1-\mathrm{N} 4$. Hence, if in addition, $\mathbf{a}, \mathbf{b} \in J$, then by Lemma 3.9, (a , b) $\in E(J, m, r)$. Consequently, in view of Lemma 4.5(i), if $(\mathbf{a}, \mathbf{b}) \in \pi$, then $\mathbf{a}, \mathbf{b} \notin J$. In particular, since $E$ is closed, $\pi$ itself is closed under E4, that is, $\pi$ is transitive.

At second, if $e \in \pi, e=\left(\alpha_{1} \cdots \alpha_{n}, \beta_{1} \cdots \beta_{n}\right)$, then all $\alpha_{i}, \beta_{i}>0$ (that is, $e$ is regular). Indeed, if for example, $\alpha_{i}>\beta_{i}=0$ for some $i$, then substitution $x_{i}^{k(J)}$ for $x_{i}$ in $e$ yields an identity contradicting Lemma 4.5(i). The set $\pi$ can be viewed therefore as an equivalence relation on the set $\Gamma_{+} \backslash J$ of those finite sequences of positive integers that are not in $J$.

Then, if $(\mathbf{a}, \mathbf{b}) \in \pi$, with $\mathbf{a}=\left(\alpha_{1}, \ldots, \alpha_{n}\right)$, and $\mathbf{b}=\left(\beta_{1}, \ldots, \beta_{t}\right)$, also the following conditions are satisfied.

$(\pi 0) \quad n=t$. 
$(\pi 1)$ Conditions N1-N4 hold for $k=k(J)$.

$(\pi 2)$ For every permutation $\sigma$ of elements of an $n$-element sequence, $(\sigma(\mathbf{a}), \sigma(\mathbf{b})) \in \pi$.

( $\pi 3)$ For every $i$ with $\alpha_{i} \neq \beta_{i},\left(\alpha_{1}, \ldots, \alpha_{n}, \min \left(\alpha_{i}, \beta_{i}\right)\right) \in J$.

$(\pi 4)$ For every $f \in \mathcal{O}$, either $(f(\mathbf{a}), f(\mathbf{b})) \in \pi$ or both $f(\mathbf{a}), f(\mathbf{b}) \in J$.

Of these conditions $\pi 0$ is trivial, $\pi 1$ is for $E \in \mathscr{I}_{S}(J, m, r), \pi 2$ is by E0, $\pi 3$ is by Lemma $4.5(\mathrm{ii})$, and $\pi 4$ follows from Lemma $4.5(\mathrm{i})$, since $(f(\mathbf{a}),(f(\mathbf{b}))$ is a consequence of $(\mathbf{a}, \mathbf{b})$.

Our structure theorem states that these conditions are also sufficient for $E(J, m, r) \cup \pi$ to be a theory.

4.8. Theorem. Given integers $m \geq 0, r>0$, and a nonempty order filter $J \subseteq[(m)]$, let $\pi$ be an equivalence relation on the set $\Gamma_{+} \backslash J$ satisfying conditions $\pi 0-\pi 4$, and let $E(J, m, r, \pi)=E(J, m, r) \cup \pi$. Then, the following hold.

(i) $E(J, m, r, \pi)$ is a closed set.

(ii) $E(J, m, r, \pi) \in \mathscr{I}_{S}(J, m, r)$, i.e., the $S$-identities in $E(J, m, r, \pi)$ are those belonging to $E(J, m, r)$.

(iii) $(\mathbf{a}, \mathbf{b}) \in E(J, m, r, \pi)$ if and only if either both $\mathbf{a}, \mathbf{b} \in J$ and satisfy conditions $\mathrm{N} 3$ and $\mathrm{N} 4$ or both $\mathbf{a}, \mathbf{b} \notin J$ and $(\mathbf{a}, \mathbf{b}) \in \pi$.

Conversely, every $E \in \mathscr{L}_{\mathrm{Com}}$ other than $E_{\varnothing}$ is of this form, i.e., if $E \in$ $\mathscr{I}_{S}(J, m, r)$, then there is the unique equivalence relation $\pi$ on the set $\Gamma_{+} \backslash J$ satisfying conditions $\pi 0-\pi 4$ such that $E=E(J, m, r, \pi)$.

Proof. We prove (i). Since $E(J, m, r)$ is a closed set, and by the definition, if $(\mathbf{a}, \mathbf{b}) \in E(J, m, r)$, then either $\mathbf{a}, \mathbf{b} \in J$ or $\mathbf{a}, \mathbf{b} \notin J$, it is enough to check the rules E0-E4 with respect to the set $\pi$ alone. We have to show that if $e=(\mathbf{a}, \mathbf{b})$ is obtained from identities in $\pi$ by applying one of E0-E4, then either $e \in E(J, m, r)$ or else $e$ satisfies conditions $\pi 0-\pi 4$. And since conditions $\pi 0-\pi 2$ are obviously satisfied (for $E \in \mathscr{I}_{S}(J, m, r)$ ), it suffices to show that either $\mathbf{a}, \mathbf{b} \in J$ or $e$ satisfies conditions $\pi 3$ and $\pi 4$.

For E0 (i.e., if $e$ is obtairied by applying E0) this is trivial. For E2 it is enough to apply $\pi 3$ and use the fact that $J$ is a filter, to see that both $\mathbf{a}, \mathbf{b} \in J$.

For E4, let $(\mathbf{a}, \mathbf{b}),(\mathbf{b}, \mathbf{c}) \in \pi$. Then by $\pi 4$, either $f(\mathbf{a}), f(\mathbf{c}) \in J$ or $(f(\mathbf{a}), f(\mathbf{b})),(f(\mathbf{b}), f(\mathbf{c})) \in \pi$. In the latter case, $(f(\mathbf{a}), f(\mathbf{c})) \in \pi$, since $\pi$ is an equivalence relation. It follows that $\pi 4$ holds for $(\mathrm{a}, \mathrm{c})$. Also $\pi 3$ does. Indeed, denote $\mathbf{c}=\left(\gamma_{1}, \ldots, \gamma_{n}\right)$. If $\alpha_{i} \leq \gamma_{i}$, then by applying $\pi 3$ for $(\mathbf{a}, \mathbf{b})$ and $(\mathbf{b}, \mathbf{c})$, either $\left(\alpha_{1}, \ldots, \alpha_{n}, \alpha_{i}\right) \in J$ (as required), or $\alpha_{i}=\beta_{i}$ and $\left(\beta_{1}, \ldots, \beta_{n}, \alpha_{i}\right) \in J$. In the latter case, by $\pi 4$, also $\left(\alpha_{1}, \ldots, \alpha_{n}, \alpha_{i}\right) \in J$. A similar argument applies for $\alpha_{i} \geq \gamma_{i}$.

For E1 and E3 the statement is simple consequence of condition $\pi 4$.

This completes the proof of (i). Now, (ii) follows from Lemma 4.6, while (iii) is immediate from Theorem 3.12 and the definition of $\Gamma_{+} \backslash J$.

For the converse, the existence of $\pi$ has been already shown. In order to show that it is unique, suppose that $E(J, m, r) \cup \pi=E(J, m, r) \cup \pi^{\prime}$. If $\pi \neq \pi^{\prime}$, then they must differ in some nontrivial identity $(\mathbf{a}, \mathbf{b})$, since trivial ones in both $\pi$ and $\pi^{\prime}$ are the same. Hence, $\mathbf{a}, \mathbf{b} \notin J$, and consequently, $(\mathbf{a}, \mathbf{b}) \notin E(J, m, r)$, which contradicts the equality assumed.

4.9. Two main questions naturally arise. The first is how to find the $E(J, m, r, \pi)$ form for a theory given by a generating set of identities; and 
the second, what the join and the meet of two theories given in $E(J, m, r, \pi)$ forms are. We answer these questions in the two next sections, respectively.

We conclude this section with some simple observations showing that, although remainders come in a great variety, they are in a sense finite, and for each theory can be described just by listing.

4.10. Given $m \geq 0, r>0$, and $\varnothing \neq J \subseteq[(m)]$, let $\mathscr{L}(J, m, r)$ denote the set of all remainders of theories in $\mathscr{I}_{S}(J, m, r)$. It is not difficult to check that $\mathscr{L}(J, m, r)$ is closed under meets and joins of equivalence relations. Hence, in view of Theorem 4.8, we have the following.

4.11. Corollary. $\mathscr{L}(J, m, r)$ is a sublattice of the lattice of partitions of the set $\Gamma_{+} \backslash J$, and $\mathscr{I}_{S}(J, m, r) \cong \mathscr{L}(J, m, r)$.

4.12. The least equivalence relation $\theta$ on the set $\Gamma_{+} \backslash J$ (i.e., that consisting of the pairs $(\mathbf{a}, \mathbf{a}))$ satisfies trivially conditions $\pi 0-\pi 4$. Hence, the least element in $\mathscr{I}_{S}(J, m, r)$ is $E(J, m, r)=E(J, m, r, \theta)$, or in other words, $E(J, m, r, \pi)$ is an $S$-theory if and only if $\pi=\theta$. Such remainders will be referred to as trivial.

On the other hand, let $\rho=\rho(J, m, r)$ denote the set of all pairs $(\mathbf{a}, \mathbf{b}) \in$ $\left(\Gamma_{+} \backslash J\right)^{2}$ such that conditions $\pi 0, \pi 1$, and $\pi 3$ are satisfied, and in addition, for all $f \in \mathscr{O}, f(\mathbf{a}) \in J$ if and only if $f(\mathbf{b}) \in J$. It is not difficult to see that this is the greatest element in $\mathscr{L}(J, m, r)$, and therefore for the greatest element $G(J, m, r)$ in $\mathscr{I}_{S}(J, m, r)$ we have

4.13. Corollary. $A$ nontrivial $e \in G(J, m, r)$ if and only if conditions $\pi 1$ and $\pi 3$ are satisfied, and in addition, for all $f \in \mathscr{O}, f(\mathbf{a}) \in J$ if and only if $f(\mathbf{b}) \in J$.

This means that every block in the partition $\rho$, and in consequence, in every partition $\pi \subseteq \rho$, is an antichain in $(\Gamma, \leq)$ all of whose elements have the same length and are in a sense equally distant from the filter $J$. Moreover, for every $n$ there is only a finite number of identities $\left(\alpha_{1} \cdots \alpha_{n}, \beta_{1} \cdots \beta_{n}\right) \in \pi$ (since $\left.\alpha_{i}, \beta_{i} \leq k(J)\right)$.

We have also the following.

4.14. Proposition. If $E=E(J, m, r, \pi)$, then there is a finite set $\pi_{0} \subseteq \pi$ such that $e \in \pi$ if and only if there is $\left(\alpha_{1} \cdots \alpha_{n}, \beta_{1} \cdots \beta_{n}\right) \in \pi_{0}$ such that $e$ is of the form (is E0-equivalent to)

$$
\left(\begin{array}{c}
\alpha_{1} \cdots \alpha_{n} \gamma_{1} \cdots \gamma_{s} \\
\beta_{1} \cdots \beta_{n} \gamma_{1} \cdots \gamma_{s}
\end{array}\right)
$$

for some $\gamma_{1}, \ldots, \gamma_{s}>0$ and $s \geq 0$, and $e \notin E(J, m, r)$. Moreover, $\gamma_{j}<$ $\min \left(\alpha_{i}, \beta_{i}\right)$ for all $j$ and $i$.

We prove this in the next section, since before we need a deeper insight into the structure of the proofs in theories of commutative semigroups.

In more detail, the remainders are considered in $\S 6$.

\section{WORD PROBLEMS}

A natural way to decide whether an identity $e$ is a consequence of set of identities $E$ is to find the form $E^{*}=E(J, m, r, \pi)$ given in Theorem 4.8. To 
describe $J$ it is enough to find its minimal elements (which up to equivalence, in view of Proposition 1.5 and Lemma 3.7, are finite in number). To describe $\pi$ it is enough, as we are going to show, to list a finite number of identities.

5.1. We shall need two additional rules of deduction, which trivially follow from E1 and E3.

$\left(\mathrm{E3}^{+}\right)$replacing $\left(\begin{array}{c}\alpha_{i} \\ \beta_{i}\end{array}\right)$ by $\left(\begin{array}{c}\alpha_{i}+1 \\ \beta_{i}+1\end{array}\right)$, for some $i$.

$\left(\mathrm{E} 1^{\times}\right)$adjoining $\left(\begin{array}{l}\gamma \\ \gamma\end{array}\right)$ column for some $\gamma \geq 1$.

It will be also convenient in the sequel to treat E0-equivalent identities as identical to avoid considering applications of the rule EO in formal proofs. Every closure below is assumed to contain all suitable trivial identities, that is, those of the form $(\mathbf{a}, \mathbf{a})$.

Now, the proof of $e$ from $E$ will be called normalized (or of type (E2, E3, $\left.\left.\mathrm{E}^{+}{ }^{+}, \mathrm{E} 1^{\times}, \mathrm{E} 4\right)\right)$, if it can be divided into five consecutive parts such that every identity in the proof, which is not already in $E$, is deduced by $\mathrm{E} 2$, whenever it occurs in the first part; by E3, whenever it occurs in the second part; by E3 ${ }^{+}$, whenever it occurs in the third part; by $E 1^{\times}$, whenever it occurs in the fourth part; and by E4, whenever it occurs in the last part. In other words, first in the proof only the rule E2 is used, then only the rule E3, etc.

\subsection{Lemma. If $e \in E^{*}$, then there is a normalized proof of $e$ from $E$.}

Proof. First we prove that there is a proof of type (E2, $\{$ E3, E1\}, E4) (i.e., using first only the rule E2, then the rules E3 and E1, and then E4).

To this end, let $C_{2}$ be the closure of $E$ under E2, and $C_{3}$ the closure of $C_{2}$ under E3 and E1. Then, $C_{3}$ consists of all matrices "greater or equal" than those in $C_{2}$ in a sense similar to that in Lemma 3.6 , that is, every column is of the form $\left(\begin{array}{l}a \\ b\end{array}\right)$ with $a=k+\sum_{i \in I} \alpha_{i}$, and $b=k+\sum_{i \in I} \beta_{i}$, where $I$ is not excluded to be empty. In particular, $C_{3}$ is clearly closed under E2, as well.

Now, let $C_{4}$ be the closure of $C_{3}$ under E4. Then, what we need to show is that $C_{4}$ is a closed set.

Obviously, $C_{4}$ is closed under E4. We show that it is closed under E1. Suppose that $(\mathbf{a}, \mathbf{b}) \in C_{4}$, and let $(\mathbf{a} 1, \mathbf{b} 1)$ denote here the result of the application of $\mathrm{E} 1$ to $(\mathbf{a}, \mathbf{b})$. Then since $C_{4}$, as a relation, is simply the transitive closure of $C_{3}$, there is a sequence $\left(\mathbf{a}_{0}, \mathbf{a}_{1}\right),\left(\mathbf{a}_{1}, \mathbf{a}_{2}\right), \ldots,\left(\mathbf{a}_{n-1}, \mathbf{a}_{n}\right)$ in $C_{3}$ such that $\mathbf{a}_{0}=\mathbf{a}$ and $\mathbf{a}_{n}=\mathbf{b}$. And since $C_{3}$ is closed under E1, $\left(\mathbf{a}_{i} 1, \mathbf{a}_{i+1} 1\right) \in C_{3}$ for all $i$. In consequence, $(\mathbf{a} 1, \mathbf{b} 1) \in C_{4}$, as required. Similarly one shows that $C_{4}$ is closed under E3 and E2 (using the fact that $C_{3}$ is so).

To complete the proof it is enough to observe that every matrix in $C_{3}$ being greater or equal to a matrix in $C_{2}$ (in the sense indicated above) can be obtained from the latter by applying first the rule E3, then the rule E3 ${ }^{+}$, and only then, if necessary, the rule $\mathrm{E} 1^{\times}$.

5.3. Now, suppose we are given a finite set of identities $E$. Then, according to Theorem $4.8, E^{*}=E(J, m, r, \pi)$ for some $J, m, r, \pi$. In $\S 5.5$ we present an algorithm to find this form. Before, we describe it in a less formal way, introducing some special notions we need, and making suitable comments. An example of application is given in $\S 5.8$.

5.4. In the first step of the algorithm, using Lemma 2.4 , we find the numbers $m, r$, and $k=k(J)$. Since, $(k) \in J$, we have $E^{*}=\left(E_{1} \cup E\left(J_{1}, m, r\right)\right)^{*}$, 
where $J_{1}=[(k)]$ is the filter generated by the sequence $(k)$, and $E_{1}$ is the set obtained from $E$ by deleting the identities with $\alpha_{i}$ or $\beta_{i} \geq k$ for some $i$. In the sequel, we call such identities $(k)$-identities.

Further, in every step of algorithm, we form a new set $E_{i}$ of identities by deleting some identities from $E_{i-1}$, and a new filter $J_{i}$ by adjoining some sequences to $J_{i-1}$ in such a way that the equation

$$
E^{*}=\left(E_{i} \cup E\left(J_{i}, m, r\right)\right)^{*}
$$

still holds. (By adjoining sequences to a filter $J$ we mean forming a new filter generated by $J$ and the adjoined sequences.) Our ultimate goal is to obtain the filter $J_{s}=J$ and a finite set $E_{s} \subseteq \pi$ determining $\pi$ in a straightforward way.

To this end, in the second step, we adjoin to the filter $J_{1}$ all the sequences corresponding to $S$-identities and irregular identities in $E_{1}$, and then, delete all these identities from $E_{1}$. The resulting pair we denote by $\left(J_{2}, E_{2}\right)$. In view of remarks in $\S 3.16$, equation (13) holds.

Now, we "separate" $J_{2}$ and $E_{2}$ in the following way. If there is an identity $(\mathbf{a}, \mathbf{b}) \in E_{2}$ such that either $\mathbf{a}$ or $\mathbf{b} \in J_{2}$, then we delete $(\mathbf{a}, \mathbf{b})$ from $E_{2}$, and adjoin $\mathbf{b}$ or a, respectively, to $J_{2}$. In view of Theorem 4.8(iii), this preserves equation (13). We repeat this until we obtain the filter $J_{3}$ and the set of identities $E_{3}$ such that $(\mathbf{a}, \mathbf{b}) \in E_{3}$ implies $\mathbf{a}, \mathbf{b} \notin J_{3}$.

To make our algorithm more efficient, we apply this procedure (whose input and output are pairs $(J, E))$ also in further steps, and it will be referred to as the separating subroutine (in short, SS).

For the third step, note that in view of condition $\pi 3$, all the sequences $\left(\alpha_{1}, \ldots, \alpha_{n}, \min \left(\alpha_{i}, \beta_{i}\right)\right)$, and $\left(\beta_{1}, \ldots, \beta_{n}, \min \left(\alpha_{i}, \beta_{i}\right)\right)$, for all $(\mathbf{a}, \mathbf{b}) \in E$, and all $i$ with $\alpha_{i} \neq \beta_{i}$, are in $J$. Hence, our third step is to adjoin all these sequences to $J_{3}$; they will be referred to as the sequences given by $\pi 3$. Then, we apply the separating subroutine. The resulting pair we denote by $\left(J_{4}, E_{4}\right)$.

Now, our idea is to apply Lemma 5.2 to determine all the consequences of the set $E_{4} \cup E\left(J_{4}, m, r\right)$. First, observe that this set is already closed under E2 (since $E\left(J_{4}, m, r\right)$ itself is a theory, and every E2-consequence of $E_{4}$, in view of the third step, is in $\left.E\left(J_{4}, m, r\right)\right)$. Hence, as the next step, we wish to find the closure under E3. Of course, it is enough to consider only E3-consequences of the set $E_{4}$ (since $E\left(J_{4}, m, r\right)$ is closed), and only those, which are not already in $E\left(J_{4}, m, r\right)$. Such consequences are finite in number (because all the sets $E_{i}$ are finite, and there is only a finite number of sequences of a fixed length not in $J$ ). We adjoin them to $E_{4}$, and after applying the separating subroutine, obtain $\left(J_{5}, E_{5}\right)$.

The fifth step is analogous, but with E3 ${ }^{+}$used instead of E3. The sixth step is also analogous, except that to keep the set $E_{i}$ finite, we adjoin only $E 1^{\times}$-consequences in no more than $s$ variables, where the number $s=s(E)$ is defined to be the maximum of $d\left(J_{4}\right)$ (defined in §3.14) and the length $n\left(E_{4}\right)$ of the longest sequence $\mathbf{a}$ in some nontrivial $(\mathbf{a}, \mathbf{b}) \in E_{4}$. The resulting pair we denote by $\left(J_{7}, E_{7}\right)$.

Now, we take the closure of $E_{7}$ under E4 (i.e., the transitive closure of $\left.E_{7}\right)$. It may happen that new $S$-identities appear. We replace these by the corresponding sequences adjoined to $J_{7}$, and to close, apply once again the separating subroutine. Then, the resulting set $E_{8} \cup E\left(J_{8}, m, r\right)$ is closed under E4, and no $S$-identity, and no identity $(\mathbf{a}, \mathbf{b})$ with $\mathbf{a}$ or $\mathbf{b}$ in $J$ is in $E_{8}$. 
Clearly, $E^{*}=\left(E\left(J_{8}, m, r\right) \cup E_{8}^{* \times}\right)^{* 4}$, where ${ }^{* \times}$ denotes the closure under $\mathrm{E} 1^{\times}$, and ${ }^{* 4}$, the closure under E4. Since $E_{8}^{* \times}$ contains no $S$-identity, all we need to show is that ${ }^{* 4}$ is superfluous, that is, that $E\left(J_{8}, m, r\right) \cup E_{8}^{* \times}$ is closed under E4.

Before, however, we put the result in a more formal way.

5.5. Algorithm to find the $E(J, m, r, \pi)$-form and solve word problems.

input $E$

1. Find $k, m$ and $r$, using Lemma 2.4 .

2. Form the filter $J$ generated by the sequence $(k)$ and all the sequences corresponding to $S$-identities and irregular identities.

Delete these identities and $(k)$-identities from $E$.

Apply $S S$ (the separating subroutine) to $(J, E)$.

3. Adjoin to $J$ all sequences given by $\pi 3$, and apply $S S$.

4. Complete $E$ with all E3-consequences.

Replace $S$-identities in $E$ by the corresponding sequences adjoined to $J$, and apply $S S$.

5. Complete $E$ with all $E 3^{+}$-consequences and apply $S S$.

6. Put $s=\max (d(J), n(E))$.

Complete $E$ with all $E 1^{\times}$-consequences in no more than $s$ variables, and apply $S S$.

7. Form the transitive closure of $E$.

Replace $S$-identities in $E$ by the corresponding sequences adjoined to $J$, and apply $S S$.

Put $\pi(E)=E$.

output $J, m, r, \pi(E)$

The main result of this section is

5.6. Theorem. Given a finite set of identities $E$, if $J, m, r, \pi(E)$ is the output of the algorithm 5.5, and ${ }^{* \times}$ denotes the closure under the rule $\mathrm{E} 1^{\times}$, then

$$
E^{*}=E(J, m, r, \pi), \quad \text { where } \pi=\pi(E)^{* \times} \backslash J^{2} .
$$

Consequently, $e=(\mathbf{a}, \mathbf{b}) \in E^{*}$ if and only if either $e$ satisfies conditions $\mathrm{N} 3-\mathrm{N} 4$ and both $\mathbf{a}, \mathbf{b} \in J$ or there is $\left(\alpha_{1} \cdots \alpha_{n}, \beta_{1} \cdots \beta_{n}\right) \in \pi(E)$ such that $e$ is of the form (is E0-equivalent to)

$$
\left(\begin{array}{c}
\alpha_{1} \cdots \alpha_{n} \gamma_{1} \cdots \gamma_{t} \\
\beta_{1} \cdots \beta_{n} \gamma_{1} \cdots \gamma_{t}
\end{array}\right)
$$

for some $\gamma_{1}, \ldots, \gamma_{t}>0$ and $t \geq 0$.

Proof. In view of our description in $\S 5.4$, and Theorem 3.12, we have only to prove that $E(J, m, r) \cup \pi(E)^{* \times}$ is closed under E4. First we make two observations.

(a) If $e \in \pi(E)^{* \times} \backslash J^{2}$ and $\alpha_{1} \geq \cdots \geq \alpha_{n}$, then $e_{1}=\left(\alpha_{1} \cdots \alpha_{s}, \beta_{1} \cdots \beta_{s}\right) \in$ $\pi(E)$, and $\alpha_{i}=\beta_{i}$ for all $i>s$. Indeed, if $e$ is an $E 1^{\times}$-consequence of some $e_{2}=\left(\alpha_{i_{1}} \cdots \alpha_{i_{s}}, \beta_{i_{1}} \cdots \beta_{i_{s}}\right) \in \pi(E)$, then in view of Step 3, all $\alpha_{i_{j}}, \beta_{i_{j}}$ in $e_{2}$ are greater than every other $\alpha_{j}$ or $\beta_{j}$ in $e$ (otherwise, a or $\mathbf{b}$ belong to $J$ ). Whence, $\left\{i_{1}, \ldots, i_{s}\right\}=\{1, \ldots, s\}$.

(b) If $e \in \pi(E)^{* *}$, then $\left(\alpha_{1}, \ldots, \alpha_{n}\right) \in J$ if and only if $\left(\beta_{1}, \ldots, \beta_{n}\right) \in J$. Indeed, suppose that $\alpha_{1} \geq \cdots \geq \alpha_{n}$ and $\left(\alpha_{1}, \ldots, \alpha_{n}\right) \in J$. Then, since 
$s \geq d(J)$, also $\left(\alpha_{1}, \ldots, \alpha_{s}\right) \in J$. Consequently, in view of remarks at the end of $\S 5.4,\left(\beta_{1}, \ldots, \beta_{s}\right) \in J$; and it follows that also $\left(\beta_{1}, \ldots, \beta_{n}\right) \in J$, as required.

Now, from (b), and since $E(J, m, r)$ is closed, we see that in order to show that $E(J, m, r) \cup \pi(E)^{* \times}$ is closed under E4, it is enough to show that if $e_{1}=\left(\alpha_{1} \cdots \alpha_{n}, \beta_{1} \cdots \beta_{n}\right)$ and $e_{2}=\left(\beta_{1} \cdots \beta_{n}, \gamma_{1} \cdots \gamma_{n}\right)$ are in $\pi(E)^{* \times} \backslash J^{2}$, then $e_{3}=\left(\alpha_{1} \cdots \alpha_{n}, \gamma_{1} \cdots \gamma_{n}\right) \in \pi(E)^{* \times}$.

In $\S 5.4$, we observed that $\pi(E)$ itself is closed under E4. In view of (a), assuming that $\beta_{1} \geq \cdots \geq \beta_{n}$, both $\left(\alpha_{1} \cdots \alpha_{s}, \beta_{1} \cdots \beta_{s}\right),\left(\beta_{1} \cdots \beta_{s}, \gamma_{1} \cdots \gamma_{s}\right) \in$ $\pi(E)$, and $\alpha_{i}=\gamma_{i}$ for $i>s$. Consequently, $\left(\alpha_{1} \cdots \alpha_{s}, \gamma_{1} \cdots \gamma_{s}\right) \in \pi(E)$, and $\left(\alpha_{1} \cdots \alpha_{n}, \gamma_{1} \cdots \gamma_{n}\right) \in \pi(E)^{* \times}$, which completes the proof.

5.7. Since by Proposition 1.2 every theory is finitely generated, we see now that the result announced in Proposition 4.14 is an immediate consequence of Theorem 5.6 and the observation (a) in the proof.

A natural equational base corresponding to the result in Theorem 5.6 is that consisting of the identities in $B(J, m, r)$ and the identities in $\pi(E)$ (which can be usually reduced so that the hypothesis of Theorem 5.6 still holds). One can observe, that if $E$ involves no more than $k$ variables, then the base in question involves no more than $k+1$ variables.

5.8. As an example, let $E$ consist of the following identities

$$
x^{4} y^{2} z^{3}=x^{2} y^{6} z^{5}, \quad x y z^{3}=x^{3} y z, \quad x^{7} y^{3} z^{3} t^{6}=x^{9} y^{5} z^{1} t^{2} .
$$

Then, as the first step, we find $k=6, m=1$, and $r=2$. There is no $S$ identity in $E$. Therefore, at the second step, $J=[(6)]$, and $E=\left\{\left(\begin{array}{c}423 \\ 265\end{array}\right),\left(\begin{array}{c}113 \\ 311\end{array}\right)\right\}$. The sequence $(265) \in J$; whence, applying SS we get $J=[(6),(423)]$ and $E=\left\{\left(\begin{array}{l}113 \\ 311\end{array}\right)\right\}$.

In Step 3, we get $J=[(6),(423),(3111), \ldots]=[(3111)]$, and $E$ without change. In Step 4, $E=\left\{\left(\begin{array}{l}113 \\ 311\end{array}\right),\left(\begin{array}{c}23 \\ 41\end{array}\right)\right\}$.

For Step 5, note that all nontrivial E3 $3^{+}$-consequences of $E$ are in $E(J, 1,2)$; whence, nothing changes in this step. For Step 6 the situation is the same (we note only that $s=\max (4,3)=4)$. And since $E$ is closed under E4, we have as a final result $J=[(3111)]$, and $\pi(E)=\left\{\left(\begin{array}{l}113 \\ 311\end{array}\right),\left(\begin{array}{c}23 \\ 41\end{array}\right)\right\}$.

In this particular case, there are only two nontrivial identities in the remainder; namely, those listed in $\pi(E)$. All other nontrivial identities belong to $E(J, 1,2)$.

5.9. Consider another example. When is the identity

$$
x_{1}^{\beta_{1}} x_{2}^{\beta_{2}} \cdots x_{s}^{\beta_{s}}=x_{1}^{\beta_{1}+1} x_{2}^{\beta_{2}} \cdots x_{s}^{\beta_{s}}
$$

$(n \geq 1)$ a consequence of the identity

$$
x_{1}^{\alpha_{1}} \cdots x_{n}^{\alpha_{n}}=x_{0} x_{1}^{\alpha_{1}} \cdots x_{n}^{\alpha_{n}}
$$

$(s \geq 1)$ in the theory of commutative semigroups?

Clearly, (15) is an $S$-identity, and therefore, for the theory generated by this identity, $J=\left[\left(\alpha_{1}, \ldots, \alpha_{n}\right)\right], m=0, r=1$, and $\pi(E)=\varnothing$. Conditions N3-N4 are in this case always satisfied. Hence, in view of Theorem 5.6, (14) is a consequence of $(15)$ if and only if the sequence $\left(\beta_{1}, \ldots, \beta_{s}\right) \in J$, that is, if and only if

$$
\left(\alpha_{1}, \ldots, \alpha_{n}\right) \leq\left(\beta_{1}, \ldots, \beta_{s}\right) .
$$


Now observe, that in view of Lemma 3.6, this condition holds if and only if $n$ items of the sizes $\alpha_{1}, \ldots, \alpha_{n}$, respectively, can be packed into $s$ bins of the sizes $\beta_{1}, \ldots, \beta_{s}$. This is the so-called BIN-PACKING problem, which is well known to be $N P$-complete (see, e.g., [10]). In consequence, we have the following.

5.10. Proposition. The problem to decide whether an identity is a consequence of a finite set of commutative semigroup identities is NP-hard.

For related results see $[23,38]$.

\section{LATtice $\mathscr{L}_{\text {Com }}$}

6.1. In order to describe the structure of the whole lattice $\mathscr{L}_{\text {Com }}$ (and thus the structure of the lattice of varieties $\mathscr{L}(\mathrm{Com}))$ we have to find the join and meet of $E_{1}=E\left(J_{1}, m_{1}, r_{1}, \pi_{1}\right)$ and $E_{2}=E\left(J_{2}, m_{2}, r_{2}, \pi_{2}\right)$. The main problem is that suggested already in Lemma 4.3. Namely, it may happen that there is a nontrivial $(\mathbf{a}, \mathbf{b}) \in \pi_{1}$ such that $\mathbf{a} \in J_{2}$, while $\mathbf{b} \notin J_{2}$. Then, $(\mathbf{a}, \mathbf{b}) \in E_{1} \vee E_{2}=$ $E\left(J_{3}, m_{3}, r_{3}, \pi_{3}\right)$, and since $\mathbf{a} \in J_{1} \cup J_{2} \subseteq J_{3}$ (cf. Lemma 4.3), it has to be, in view of Theorem 4.8(iii), $\mathrm{b} \in J_{3} \backslash J_{1} \cup J_{2}$. Hence, $J_{3} \neq J_{1} \cup J_{2}$.

For $E_{1}, E_{2}$ as above, we define $J_{u}$ to be the least filter $J \supseteq J_{1} \cup J_{2}$ such that whenever $(\mathbf{a}, \mathbf{b}) \in \pi_{1} \cup \pi_{2}$ and either $\mathbf{a}$ or $\mathbf{b}$ is in $J_{1} \cup J_{2}$, then both $\mathbf{a}, \mathbf{b} \in J$. Clearly, $J_{3} \supseteq J_{u}$, and we are going to show that actually the equality holds.

It is useful, however, to have a more direct definition of $J_{u}$. Given a set $J$ and an equivalence relation $\pi$, let $C(J, \pi)$ be the union of all those blocks in $\pi$, which have a point in common with $J$, completed with all equivalent sequences with zeros (i.e., $\mathbf{a} \in C(J, \pi)$ if for the sequence $\mathbf{a}_{0}$ obtained from $\mathbf{a}$ by deleting all zeros there is $\mathbf{b} \in J$ such that $(\mathbf{a}, \mathbf{b}) \in \pi)$. Then, it is easy to observe, that $J_{u}=J_{1} \cup J_{2} \cup C$, where $C=C\left(J_{1} \cup J_{2}, \pi_{1} \vee \pi_{2}\right)$, and $\vee$ denotes the join of equivalence relations.

Below, we use $E^{S}$ to denote the Schwabauer part of the theory $E$ (i.e., for $\left.E=E(J, m, r, \pi), E^{S}=E(J, m, r)\right)$, and with superscripts we denote cartesian powers. Then, we have the following.

6.2. Theorem. If $E_{1}=E\left(J_{1}, m_{1}, r_{1}, \pi_{1}\right)$, and $E_{2}=E\left(J_{2}, m_{2}, r_{2}, \pi_{2}\right) \in$ $\mathscr{L}_{\text {Com }}$, then the following hold.

(i) $E_{1} \supseteq E_{2}$ if and only if $J_{1} \supseteq J_{2}, m_{1} \leq m_{2}, r_{1}$ divides $r_{2}$, and $\pi_{1} \cup J_{1}^{2} \supseteq$ $\pi_{2}$.

(ii) $E_{1} \vee E_{2}=E\left(J_{1} \cup J_{2} \cup C, \min \left(m_{1}, m_{2}\right), \operatorname{gcd}\left(r_{1}, r_{2}\right), \pi_{1} \vee \pi_{2} \backslash C^{2}\right)$, where $C=C\left(J_{1} \cup J_{2}, \pi_{1} \vee \pi_{2}\right)$.

(iii) $E_{1} \cap E_{2}=E\left(J_{1} \cap J_{2}, \max \left(m_{1}, m_{2}\right), \operatorname{lcm}\left(r_{1}, r_{2}\right), \pi\right)$, where $\pi=\left(\pi_{1} \cap\right.$ $\left.\pi_{2}\right) \cup\left(\pi_{1} \cap E_{2}^{S}\right) \cup\left(\pi_{2} \cap E_{1}^{S}\right)$.

Moreover, if $E_{i}=E\left(J_{i}, m_{i}, r_{i}, \pi_{i}\right)$ is a family of theories in $\mathscr{L}_{\text {Com }}$, then

(iv) $\bigvee E_{i}=E\left(\bigcup J_{i} \cup C, \min \left\{m_{i}\right\}, \operatorname{gcd}\left\{r_{i}\right\}, \bigvee \pi_{i} \backslash C^{2}\right)$, where $C=$ $C\left(\bigcup J_{i}, \bigvee \pi_{i}\right)$.

(v) $\cap E_{i}=E\left(\bigcap J_{i}, \max \left\{m_{i}\right\}, \operatorname{lcm}\left\{r_{i}\right\}, \cap\left(\pi_{i} \cup E_{i}^{S}\right) \backslash \cap E_{i}^{S}\right)$, provided $\cap J_{i}$ is nonempty and both $\max \left\{m_{i}\right\}$ and $\operatorname{lcm}\left\{r_{i}\right\}$ exist; if not, $\cap E_{i}=E_{\varnothing}$.

Proof. We use Theorem 3.12. For (i), the "only if" part is trivial. 
For the "if" part it is enough to show that if $(\mathbf{a}, \mathbf{b}) \in \pi_{2} \subseteq \pi_{1} \cup J_{1}^{2}$, then $(\mathbf{a}, \mathbf{b}) \in E_{1}$. If $(\mathbf{a}, \mathbf{b}) \in \pi_{1}$, then the result is by the definition of $E_{1}$. If $(\mathbf{a}, \mathbf{b}) \in J_{1}^{2}$, then since it satisfies condition $\pi 1,(\mathbf{a}, \mathbf{b}) \in E\left(J_{1}, m_{1}, r_{1}\right) \subseteq E_{1}$, as required.

For (ii), let us denote $J=J_{1} \cup J_{2} \cup C$. First we show that

$$
E_{3}=E\left(J, \min \left(m_{1}, m_{2}\right), \operatorname{gcd}\left(r_{1}, r_{2}\right), \pi\right)
$$

with $\pi=\pi_{1} \vee \pi_{2} \backslash C^{2}$ is a theory, that is, satisfies the conditions of Theorem 4.8. Since $E_{1} \in \mathscr{I}_{N}\left(k\left(J_{1}\right), m_{1}, r_{1}\right), E_{2} \in \mathscr{I}_{N}\left(k\left(J_{2}\right), m_{2}, r_{2}\right)$, we have, by virtue of Lemma 2.4, $m(J) \geq \min \left(m_{1}, m_{2}\right)$. Hence, it is enough to check that $\pi=\pi_{1} \vee \pi_{2} \backslash C^{2}$ is an equivalence relation on the set $\Gamma_{+} \backslash J$ satisfying conditions $\pi 0-\pi 4$. Of these, $\pi 0-\pi 3$ are easy to check. We check $\pi 4$.

First note that if $(\mathbf{a}, \mathbf{b}) \in \pi_{1} \cup \pi_{2}$ and for some $f \in \mathscr{O}$ one of $f(\mathbf{a})$ or $f(\mathbf{b})$ is in $J$, then both $f(\mathbf{a}), f(\mathbf{b}) \in J$. This is immediate, if (according to $\pi 4)$ both $f(\mathbf{a}), f(\mathbf{b}) \in J_{1}$ or $J_{2}$, and follows from the definition of $J$, if $(f(\mathbf{a}), f(\mathbf{b})) \in \pi_{1} \cup \pi_{2}$.

Now, if $(\mathbf{a}, \mathbf{b}) \in \pi_{1} \vee \pi_{2} \backslash C^{2}$, then there is a sequence

$$
\left(\mathbf{a}, \mathbf{a}_{1}\right),\left(\mathbf{a}_{1}, \mathbf{a}_{2}\right), \ldots,\left(\mathbf{a}_{n}, \mathbf{b}\right)
$$

such that each element is in $\pi_{1}$ or $\pi_{2}$. Whence, if $f\left(\mathbf{a}_{i}\right) \in J$ for at least one $i$, then by the remark above both $f(\mathbf{a}), f(\mathbf{b}) \in J$, as required. Otherwise, by $\pi 4$, all elements in the sequence $\left(f(\mathbf{a}), f\left(\mathbf{a}_{1}\right)\right),\left(f\left(\mathbf{a}_{1}\right), f\left(\mathbf{a}_{2}\right)\right), \ldots,\left(f\left(\mathbf{a}_{n}\right), f(\mathbf{b})\right)$ are in $\pi_{1}$ or in $\pi_{2}$. Consequently, $f(\mathbf{a}), f(\mathbf{b}) \in \pi_{1} \vee \pi_{2} \backslash C^{2}$, proving the property $\pi 4$, and thus showing that $E_{3}$ is a theory.

Now, by (i), $E_{3} \supseteq E_{1}, E_{2}$, and consequently, $E_{3} \supseteq E_{1} \cup E_{2}$. On the other hand, by the argument in $\S 6.1$,

$$
E\left(J, \min \left(m_{1}, m_{2}\right), \operatorname{gcd}\left(r_{1}, r_{2}\right)\right) \subseteq E_{1} \vee E_{2},
$$

and obviously, $\pi_{1} \vee \pi_{2} \subseteq E_{1} \vee E_{2}$. This completes the proof of (ii).

For (iii), compute

$E_{1} \cap E_{2}=\left(E_{1}^{S} \cup \pi_{1}\right) \cap\left(E_{2}^{S} \cup \pi_{2}\right)=\left(E_{1}^{S} \cap E_{2}^{S}\right) \cup\left(E_{1}^{S} \cap \pi_{2}\right) \cup\left(E_{2}^{S} \cap \pi_{1}\right) \cup\left(\pi_{1} \cap \pi_{2}\right)$.

Hence, by Theorems 3.12 and 4.8,

$E_{1} \cap E_{2}=E\left(J_{1} \cup J_{2}, \max \left(m_{1}, m_{2}\right), \operatorname{lcm}\left(r_{1}, r_{2}\right),\left(E_{1}^{S} \cap \pi_{2}\right) \cup\left(E_{2}^{S} \cap \pi_{1}\right) \cup\left(\pi_{1} \cap \pi_{2}\right)\right)$.

By Lemma 4.3 and in view of the description of the equivalence relation $\pi$ in $\S 4.7$, this is the required form $E(J, m, r) \cup \pi$.

The proofs of (iv) and (v) are analogous and are left to the reader.

6.3. For applications, it is useful to note the following. If $J_{1} \supseteq J_{2}$, then formulations in (ii) and (iii) simplify to

(ii) ${ }^{*} \quad E_{1} \vee E_{2}=E\left(J_{1}, \min \left(m_{1}, m_{2}\right), \operatorname{gcd}\left(r_{1}, r_{2}\right), \pi_{1} \vee \pi_{2} \backslash J_{1}^{2}\right)$,

(iii) $^{*} \quad E_{1} \cap E_{2}=E\left(J_{2}, \max \left(m_{1}, m_{2}\right), \operatorname{lcm}\left(r_{1}, r_{2}\right), \pi_{2} \cap\left(\pi_{1} \cup E_{1}^{S}\right)\right)$.

6.4. Using the formulas above for $E=E(J, m, r, \pi)$ and $k=k(J)$, we obtain the following identity.

$$
E=(E \vee E(k, 0,1)) \cap E(1,0, r) \cap E(m, m, 1) .
$$

Apply it to varieties. If $\mathrm{Nil}_{k}=\mathscr{V}(k, 0,1)$ is the variety of nil-semigroups defined by $x^{k}=0, \mathscr{A}_{r}=\mathscr{V}(1,0, r)$ is the variety of abelian groups defined 
by $x^{r}=1$, and $\mathrm{Ap}_{m}=\mathscr{V}(m, m, 1)$ is the variety of aperiodic semigroups defined by $x^{m+1}=x^{m}$, then, for a variety $\mathscr{V}=\mathscr{V}(J, m, r, \pi)$ corresponding to the equational theory $E=E(J, m, r, \pi)$, we have

$$
\mathscr{V}=\left(\mathscr{V} \cap \mathrm{Nil}_{k}\right) \vee \mathscr{A}_{r} \vee \mathrm{Ap}_{m}
$$

a result proved in [24].

6.5. Another important result from [24] also easily follows. The mapping

$$
\phi: \mathscr{V}(J, m, r, \pi) \rightarrow(\mathscr{V}(J, 0,1, \pi), m, r)
$$

of $\mathscr{L}(\mathrm{Com})$ into the cartesian product of $\mathscr{L}(\mathrm{Nil}) \times N_{0} \times N_{1}$ (where $N_{0}$ and $N_{1}$ are the natural numbers with and without zero, ordered naturally and by division, respectively) is an embedding of meet-semilattices. This is straightforward from Theorem 6.2(ii).

In contrast to [24], however, one can see explicitly why joins (resp., equational theory meets) are not preserved ( $\pi$ in (iii) depends on $m_{i}$ and $r_{i}$ ), and an explicit counterexample can be easily given.

Let $\bar{\phi}: E(J, m, r, \pi) \rightarrow(E(J, 0,1, \pi), m, r)$ be the map dual to $\phi$. Let $E_{1}=E(1,0,2)$, and $E_{2}=\left(J_{(211)}, 0,1, \pi\right)$, where $J_{(211)}$ is the filter generated by the sequence $(2,1,1)$, and $\pi$ is the remainder generated by the identity $x^{2} y=x y^{2}$. Then, by (iii) ${ }^{*}, E_{1} \cap E_{2}=E\left(J_{(211)}, 0,2\right)$ (since $\left.x^{2} y=x y^{2} \notin E_{1}\right)$, while $\bar{\phi}\left(E_{1}\right)=(E(1,0,1), 0,2), \bar{\phi}\left(E_{2}\right)=\left(E_{2}, 0,1\right)$, and $\bar{\phi}\left(E_{1}\right) \cap \bar{\phi}\left(E_{2}\right)=\left(E_{2}, 0,2\right) \neq \bar{\phi}\left(E_{1} \cap E_{2}\right)$.

We proceed to describe the greatest element in the interval $\mathscr{I}_{S}(J, m, r)$. This will shed more light on the structure of the remainders.

6.6. Lemma. Let $E=E(J, m, r, \pi)$. If $e \in \pi$ is of the form (1) and $\alpha_{1} \geq$ $\alpha_{2} \geq \cdots \geq \alpha_{n}$, then $\alpha_{i}=\beta_{i}$ for all $i \geq d(J)$.

Proof. Suppose that for some $j \geq d(J), \alpha_{j}<\beta_{j}$. Then, by $\pi 3$, the sequence $\left(\alpha_{1}, \ldots, \alpha_{j}, \alpha_{j}, \alpha_{j+1}, \ldots, \alpha_{n}\right) \in J$. It follows that there is $\mathbf{a}$ in the fundamental antichain of $J$ such that $\mathbf{a} \leq\left[\alpha_{1}, \ldots, \alpha_{j}, \alpha_{j}, \alpha_{j+1}, \ldots, \alpha_{n}\right]$. By assumption and since the length of $\mathbf{a}$ is less or equal to $d(J) \leq j$, it follows in view of Lemma 3.6, that $\mathbf{a} \leq\left[\alpha_{1}, \ldots, \alpha_{j}\right]$. Consequently, $\left(\alpha_{1}, \ldots, \alpha_{n}\right) \in J$, a contradiction.

Note that the result in Lemma 6.6 does not mean that $\pi$ is determined by the identities in at most $n_{0}=d(J)$ variables. Generally, an integer $n_{0}$ with this property always exists, but may be arbitrary large and does not depend on $J$ at all. In the special case of $\rho$ we have the following.

6.7. Proposition. Let $G(J, m, r)=E(J, m, r, \rho), e \in \rho$, and assume that the least number among all $\alpha_{i}$ and $\beta_{i}$ is $\alpha_{n}$. Denote $n_{0}=(k(J)-1)(d(J)-1)$. If $e$ is nontrivial and $n>n_{0}$, then $\left(\alpha_{1} \cdots \alpha_{n-1}, \beta_{1} \cdots \beta_{n-1}\right) \in \rho$ and $\alpha_{n}=\beta_{n}$.

In particular, $G(J, m, r)$ has a basis involving no more than $n_{0}$ variables.

Proof. Denote $(\mathbf{a}, \mathbf{b})=\left(\alpha_{1} \cdots \alpha_{n-1}, \beta_{1} \cdots \beta_{n-1}\right)$. By Lemma 6.6, since $e$ is nontrivial, $k(J), d(J)>1$ and $\alpha_{n}=\beta_{n}>0$.

By virtue of Corollary 4.13, (a, b) satisfies condition $\pi 1$ (for N1 and N2 note that $n \geq k(J))$. An argument similar to that in the proof of Lemma 6.6 shows that $(\mathbf{a}, \mathbf{b})$ also satisfies $\pi 3$.

For the remaining condition suppose, on the contrary, that $f(\mathbf{a}) \in J$ and $f(\mathbf{b}) \notin J$ for some $f \in \mathscr{O}$, and denote by $f(\mathbf{b}) \beta_{n}$ the sequence obtained from 
$f(\mathbf{b})$ by adjoining $\beta_{n}$. Then, since $f(\mathbf{a}) \in J, f(\mathbf{a}) \alpha_{n} \in J$, and since $e \in \rho$, also $f(\mathbf{b}) \beta_{n} \in J$. Since $\beta_{n}=\alpha_{n}$ is minimal, it follows that the length of $f(\mathbf{b})$ is less than $d(J)$ (otherwise, similarly as in the proof of Lemma 6.6, $f(\mathbf{b}) \in J)$. Since $\sum f(\mathbf{b})>n_{0}$, by the pigeonhole principle one of the elements of the sequence $f(\mathbf{b})$ must be not less than $k(J)$. Whence, $f(\mathbf{b}) \in J$, a contradiction.

Now, let $G$ be the set of all identities in $\rho(J, m, r)$ in at most $n_{0}$ variables. In view of the remarks following Corollary $4.13, G$ is finite. The result in Proposition 6.7 means that every identity in $\rho$, and therefore every identity in $\pi \subseteq \rho$, is an $\mathrm{E} 1^{\times}$-consequence of some member of $G$. In particular, in view of Proposition 4.14 , every $\pi$ is $E 1^{\times}$-generated by a finite number of $E 1^{\times}$consequences of $G$. Although this representation is not unique, in many cases it helps to determine completely the interval $\mathscr{I}_{S}(J, m, r)$.

We conclude this section with a number of examples establishing some simple facts and illustrating how varied the remainders and the intervals $\mathscr{I}_{S}(J, m, r)$ can be.

Example 1. Let $J$ be any filter generated by sequences of the length at most 2. Then, by Lemma 6.6 , for every $E=E(J, m, r, \pi), \pi$ is trivial, and consequently, $\mathscr{I}_{S}(J, m, r)=\{E(J, m, r)\}$ has precisely one element.

Example 2. Suppose that $(m, 1,1, \ldots, 1) \in J$, where $m>0$, and 1 occurs $s>0$ times. Then, every identity $e$ with $n>s$ satisfying N1-N4 is in $E(J, m, r)$. Whence, $\rho=\rho(J, m, r)$ consists only of a finite number of nontrivial identities, and consequently, $\mathscr{I}_{S}(J, m, r)$ is finite, as well. Obviously, for every $J$ with this property, $\mathscr{I}_{S}(J, m, r)$ can be effectively described. The problem with a uniform description of these intervals is that they are as varied as possible. In fact, every finite lattice can be embedded into one of these intervals, as the next example shows.

Example 3. Let $J=[(1,1, \ldots, 1)]$ with 1 occurring $s+1$ times and, to simplify the example, let $m=r=1$. Then, by Corollary 4.13 , in particular, every identity of the form $x^{u} y^{s-u}=x^{w} y^{s-w} \quad(0<u, w<s)$ is in $\rho(J, m, r)$. Note, that every nontrivial consequence of any of these identities is in $E(J, m, r)$; so they are independent. Now, to every partition of the set of terms $\left\{x^{1} y^{s-1}, x^{2} y^{s-2}, \ldots, x^{t} y^{s-t}\right\}$ (with $t \leq s / 2$ ) we assign the theory $E(J, m, r, \pi)$, where $\pi$ is generated by identities corresponding to the pairs in the blocks of the partition. Clearly, such theories form a sublattice of the lattice $\mathscr{I}_{S}(J, m, r)$ isomorphic to the partition lattice $\Pi_{t}$.

This is, in fact, precisely the result of [4].

Example 4. If $\rho=\rho(J, m, r)$ contains at least one nontrivial identity $\left(\alpha_{1} \cdots \alpha_{n}, \beta_{1} \cdots \beta_{n}\right)$ with $n>d(J)$, then $\mathscr{I}_{S}(J, m, r)$ is infinite. Indeed, in such a case every identity of the form $(\mathbf{a}, \mathbf{b})$, where

$$
\mathbf{a}=\left(\alpha_{1}, \ldots, \alpha_{n}, 1,1, \ldots, 1\right) \text { and } \mathbf{b}=\left(\beta_{1}, \ldots, \beta_{n}, 1,1, \ldots, 1\right),
$$

is in $\rho$, and all these identities (together with the $S$-identities corresponding to $J$ ) generate an infinite descending chain of theories in $\mathscr{I}_{S}(J, m, r)$.

If, in addition, all integers occurring in the sequences belonging to the fundamental antichain of $J$ are greater than one, then the construction above also 
shows that in such a case $\mathscr{I}_{S}(J, m, r)$ has no atoms. On the other hand, by Proposition 1.2, $\mathscr{I}_{S}(J, m, r)$ has at least one coatom; and by a result of Almeida [2] mentioned in the introduction, the number of coatoms (atoms) in $\mathscr{I}_{S}(J, m, r)$ is always finite.

Example 5. Consider the sublattice $\mathscr{L}(\mathscr{N})$ of $\mathscr{L}$ (Com), of varieties of nilpotent commutative semigroups. It is the union of the ascending chain of the lattices $\mathscr{L}\left(\mathscr{N}_{k}\right)$ of subvarieties of the variety $\mathscr{N}_{k}$ of commutative semigroups defined by the law $x_{1} \cdots x_{k}=0$. The equational theory of $\mathscr{N}_{k}$ is $E\left(\mathscr{N}_{k}\right)=$ $E\left(J_{k}, 0,1\right)$, where $J_{k}=[(1,1, \ldots, 1)]$ with 1 occurring $k$ times.

There are only finitely many sequences $\left(\alpha_{1}, \ldots, \alpha_{n}\right)$ with $\sum \alpha_{i}<k$, and therefore the lattices $\mathscr{L}\left(\mathscr{N}_{k}\right)$ are all finite. Thus, every proper interval of $\mathscr{L}\left(\mathscr{N}_{k}\right)$ is finite, and can be explicitly described, using Theorem 6.2 and Proposition 6.7.

Example 6. If $E=E(J, m, r, \pi)$ and a nontrivial $e \in \pi$, then by conditions $\pi 0$ and $\pi 4, e$ is regular, and $\left(\alpha_{1}, \ldots, \alpha_{n}\right),\left(\beta_{1}, \ldots, \beta_{n}\right)$ are noncomparable in $(\Gamma, \leq)$. It is not difficult to see that every identity $e$ satisfying these conditions is a member of the remainder $\pi$ of some theory $E$.

Indeed, let for example, $e: x^{2} y z^{5}=x^{3} y^{3} z \quad(\mathbf{a}=(2,1,5), \mathbf{b}=(3,3,1))$. We construct a filter $J$ such that $f(\mathbf{a}), f(\mathbf{b}) \in J$ for all nontrivial $f \in \mathscr{O}$. There is a unique such filter; namely, in this case, $J=[(2,1,5,1),(3,3,1,1)]$. Then, $e$ satisfies obviously conditions $\pi 0-\pi 4$, and for instance, $e \in \rho(J, 1,1)$.

This construction works for any regular identity with noncomparable sequences of the exponents, and may be used to construct various special examples. In fact, it was used to construct the counterexample in $\S 6.5$.

\section{FINITE SEMIGROUPS}

In this section we apply our results to finite semigroups and finitely generated varieties. First we introduce some notation.

7.1. Given a commutative semigroup $\mathbf{S}$ and a variety $\mathscr{V}$, by $E(\mathbf{S})$ and $E(\mathscr{V})$ we denote the equational theories of $\mathbf{S}$ and $\mathscr{V}$, respectively. If $E(\mathbf{S})=$ $E(J, m, r, \pi)$, then we denote $k(\mathbf{S})=k(J), m(\mathbf{S})=m$, and $r(\mathbf{S})=r$. Similarly, we define $k(\mathscr{V}), m(\mathscr{V})$, and $r(\mathscr{V})$ for $E(\mathscr{V})=E(J, m, r, \pi)$.

Note, that for a given finite $\mathbf{S}$, using the definitions in $\S 2.2$, all these parameters can be easily computed. Below, we shall see, in particular, how other parameters of $E(\mathbf{S})$ can be computed.

We start from the following.

7.2. Lemma. Let $E \in \mathscr{I}_{N}(k, m, r)$ and let an identity

$$
x_{1}^{m} x_{2} \cdots x_{n}=x_{1}^{m+r} x_{2} \cdots x_{n}
$$

be in $E$ for some $n \geq 1$. Then,

(i) for $m>0$, every nontrivial identity in at least $n$ variables satisfying conditions N1-N4 is in $E$,

(ii) for $m=0$, every nontrivial identity satisfying conditions $\mathrm{N} 1-\mathrm{N} 4$ and such that at least $n-1$ variables have occurrences on both the sides is in $E$. 
Proof. If $e$ is a nontrivial identity satisfying the conditions of (i), then both $\left(\alpha_{1}, \ldots, \alpha_{n}\right),\left(\beta_{1}, \ldots, \beta_{n}\right)$ are in the filter generated by $(m, 1, \ldots, 1)$, with 1 occurring $n-1$ times. Applying Theorem 3.12 yields the desired result. A similar argument proves (ii).

7.3. Proposition. Let $\mathbf{S}$ be a finitely generated commutative semigroup with $q$ generators. If $k(\mathbf{S})=k$ and $m(\mathbf{S})=m$, then the identity (20) holds in $\mathbf{S}$ for every $n \geq q(k-1)-m+2$. In consequence, conditions (i) and (ii) of Lemma 7.2 hold for $E(\mathbf{S})$.

Proof. Let $a_{1}, a_{2}, \ldots, a_{n}$ be arbitrary elements of $\mathbf{S}$. Representing each $a_{i}$ as a product of generators $g_{i}$, we get

$$
a_{1}^{m} a_{2} \cdots a_{n}=g_{1}^{\alpha_{1}} \cdots g_{q}^{\alpha_{q}}
$$

for some $\alpha_{1}, \ldots, \alpha_{q} \geq 0$ with $\sum \alpha_{i} \geq m+n-1 \geq q(k-1)+1$. It follows that $\alpha_{i} \geq k$ for some $i$. Whence, we may assume that

$$
a_{1}^{m} a_{2} \cdots a_{n}=g_{1}^{\alpha_{1}} \cdots g_{s}^{\alpha_{s}} g_{s+1}^{\alpha_{s+1}} \cdots g_{q}^{\alpha_{q}}
$$

where $\alpha_{1} \geq k, \alpha_{i} \geq m$ for $i \leq s$, and $\alpha_{i}<m$, otherwise $(1 \leq s \leq q)$. Then, in view of $(8)$,

$$
a_{1}^{m+r} a_{2} \cdots a_{n}=g_{1}^{\beta_{1}} \cdots g_{s}^{\beta_{s}} g_{s+1}^{\alpha_{s+1}} \cdots g_{q}^{\alpha_{q}},
$$

where $\beta_{1} \geq k, \beta_{i} \geq m$ for $i \leq s$, and $\beta_{i} \equiv \alpha_{i}(\bmod r)$, where $\left.r=r(\mathbf{S})\right)$. Hence, by (8),

and the result follows.

$$
a_{1}^{m} a_{2} \cdots a_{n}=a_{1}^{m+r} a_{2} \cdots a_{n}
$$

It follows from the proposition above that if $E(\mathbf{S})=E(J, m, r, \pi)$, then $[(m, 1, \ldots, 1)] \subseteq J \subseteq[(m)]$ with 1 occurring $q(k-1)-m+1$ times. There are only finitely many filters with this property, and therefore, for finite $\mathbf{S}, J$ can be effectively computed without appeal to Theorem 5.6. To compute $\pi$ the next proposition applies.

7.4. Proposition. If a semigroup $\mathbf{S}$ has $q$ generators, $k(\mathbf{S})=k$ and $m(\mathbf{S})=$ $m$, then $\mathbf{S}$ has a basis for its identities involving (besides $x(y z)=(x y) z, x y=$ $y x)$ no more than $n=q(k-1)-m+2$ variables.

Proof. If $m>0$, then the result is a simple consequence of Proposition 7.3. Hence, suppose that $m=0$ and let

$$
x_{1}^{\alpha_{1}} \cdots x_{s}^{\alpha_{s}} w=z_{1}^{\beta_{1}} \cdots z_{t}^{\beta_{t}} w
$$

be an identity holding in $\mathbf{S}$, where $w$ is a word, and $x_{1}, \ldots, x_{s}, z_{1}, \ldots, z_{t}$ are pairwise distinct variables not occurring in $w$. We wish to show that this is a consequence of the set $I d_{n}(\mathbf{S})$ of identities in at most $n$ variables, holding in $\mathbf{S}$.

Obviously, $r=r(\mathbf{S})$ divides both $\alpha_{i}$ and $\beta_{i}$ for all $i$, and we may assume that $s>0$, and $t \geq 0$ (because, if both $s=t=0$, then (21) either is in $I d_{n}(\mathbf{S})$, or by Lemma 7.2(ii), is a consequence of $\left.I d_{n}(\mathbf{S})\right)$.

First we need some further identities holding in $\mathbf{S}$. Substituting $x_{i}=y^{k}$ in (21), for all $i$, and applying (3), which is in $I_{n}(\mathbf{S})$, we get

$$
y^{k r} w=z_{1}^{\beta_{1}} \cdots z_{t}^{\beta_{t}} w .
$$


Comparing it with $(21)$ yields

$$
y^{k r} w=x_{1}^{\alpha_{1}} \cdots x_{s}^{\alpha_{s}} w
$$

Applying (8) to (22) and (23) yields

$$
\begin{aligned}
& y^{k r} z_{1}^{\beta_{1}} \cdots z_{t}^{\beta_{t}} w=z_{1}^{\beta_{1}} \cdots z_{t}^{\beta_{t}} w, \\
& y^{k r} x_{1}^{\alpha_{1}} \cdots x_{s}^{\alpha_{s}} w=x_{1}^{\alpha_{1}} \cdots x_{s}^{\alpha_{s}} w .
\end{aligned}
$$

Now, let $o(w)$ denote the number of variables occurring in $w$. If $s+o(w) \geq$ $n-1$, then $(25)$ is a consequence of $I d_{n}(\mathbf{S})$, by Lemma 7.2(ii). Otherwise, (25) is among $I d_{n}(\mathbf{S})$. The same concerns (24).

From (24) and (25), by (8) (which is in $I d_{n}(\mathbf{S})$ ), we get (22) and (23), and these yield (21). Hence, $(21)$ is a consequence of $I d_{n}(\mathbf{S})$, as required.

The proposition above improves Theorem 16 in [27], where the same was proved for $n=2 q k+1$. Moreover, our bound is the best possible, as the following example shows.

7.5. Let $\mathscr{V}$ be a theory of nil-semigroups defined by $x^{k}=0$, and $\mathbf{F}_{q}=$ $\mathbf{F}_{q}(\mathscr{V})$, the $\mathscr{V}$-free semigroup on $q$ free generators. Then, $E\left(\mathbf{F}_{q}\right) \in \mathscr{J}_{N}(k, 0,1)$, and no identity

$$
x_{1} x_{2} \cdots x_{s}=x_{2} \cdots x_{s}
$$

with $s<n=q(k-1)+2$ holds in $\mathbf{F}_{q}$. Indeed, suitably substituting powers of generators $g_{1}, \ldots, g_{q}$ for $x_{1}, \ldots, x_{s}$ would yield

$$
g_{1}^{k} g_{2}^{k-1} \cdots g_{q}^{k-1}=g_{2}^{k-1} \cdots g_{q}^{k-1}
$$

which is not true in $\mathbf{F}_{q}$ (see Lemma 2.8). Similarly, one can prove that no nontrivial identity in less than $n$ variables such that each variable occurs at most once on each side holds in $\mathbf{F}_{q}$. Using this, it is not difficult to show that (20), which holds in $\mathbf{F}_{q}$ by Proposition 7.3 , is not a consequence of $I d_{n-1}\left(\mathbf{F}_{q}\right)$.

7.6. Recall that a variety $\mathscr{V}$ is said to be finitely generated if it is generated by a finite algebra (or equivalently, by a finite number of finite algebras). We prove the following.

7.7. Theorem. Let $\mathscr{V}$ be a variety of commutative semigroups with $m(\mathscr{V})=m$ and $r(\mathscr{V})=r$. Then, $\mathscr{V}$ is finitely generated if and only if the identity (20) is in $E(\mathscr{V})$ for some $n \geq 2$.

Proof. The "only if" part follows trivially from Proposition 7.3. For the "if" part, note that if $m>0$, then, by Proposition 7.3 , the $\mathscr{V}$-free semigroup $\mathbf{F}_{n}=\mathbf{F}_{n}(\mathscr{V})$ on $n$ free generators generates $\mathscr{V}$. We show that the same is true for $m=0$.

Since the identities in at most $n$ variables holding in $\mathbf{F}_{n}$ are precisely those belonging to $E(\mathscr{V})$, it is enough to show that every identity $e$ holding in $\mathbf{F}_{n}$ is a consequence of the set $I d_{n}\left(\mathbf{F}_{n}\right)$.

Suppose that $e$ is given in the form of (21). If the number of variables $o(w)$ occurring in $w$ is not less than $n-1$, then the result is by Lemma 7.2(ii). Hence, assume that $o(w)=n-p$ with $p>1$.

Let $v=x_{1}^{\alpha_{1}} \cdots x_{p-1}^{\alpha_{p-1}} x_{p}^{\alpha}$ be the word obtained from $x_{1}^{\alpha_{1}} \cdots x_{s}^{\alpha_{s}}$ by identification of variables with indices greater than $p-1$, whenever $s>p$, and $v=x_{1}^{\alpha_{1}} \cdots x_{s}^{\alpha_{s}}$, otherwise. Analogously define the word $u$ for $y_{1}^{\beta_{1}} \cdots y_{t}^{\beta_{t}}$. 
Then, $v w=u w$ is a consequence of $(21)$, and therefore is in $E\left(\mathbf{F}_{n}\right)$ (note, that $o(v w), o(u w) \leq n-1)$. Also, $x^{\alpha} w=y^{\beta} w$, obtained from (21) by identifications of all variables $x_{i}$ and all variables $y_{i}$, respectively, is in $E\left(\mathbf{F}_{n}\right)$. Moreover, identifying separately $x$ 's and $y$ 's, we get that all $x_{1}^{\alpha_{1}} \cdots x_{s}^{\alpha_{s}} w=$ $v w=x^{\alpha} w$, and $y_{1}^{\beta_{1}} \cdots y_{t}^{\beta_{t}} w=u w=y^{\beta} w$, are in $E\left(\mathbf{F}_{n}\right)$.

Now, by the assumption, the number of variables occurring in $x^{\alpha} w=y^{\beta} w$ does not exceed $n$. The same is true for $v w=x^{\alpha} w$. Further, by Lemma 7.2(ii) (or because it is trivial), $x_{1}^{\alpha_{1}} \cdots x_{s}^{\alpha_{s}} w=v w$ is a consequence of $I_{n}\left(\mathbf{F}_{n}\right)$. Hence, $x_{1}^{\alpha_{1}} \cdots x_{s}^{\alpha_{s}} w=x^{\alpha} w$ is also a consequence $I d_{n}\left(\mathbf{F}_{n}\right)$. Similarly, $y_{1}^{\beta_{1}} \cdots y_{t}^{\beta_{t}}$ $=y^{\beta} w$ is a consequence of $I d_{n}\left(\mathbf{F}_{n}\right)$, and we conclude that so is (21).

The join of two finitely generated varieties is, of course, finitely generated, as well. From the theorem above, it follows that the intersection of two finitely generated varieties of commutative semigroups is also finitely generated. Hence, we have the following.

7.8. Corollary. The set $\mathscr{L}\left(\mathrm{Com}_{F}\right)$ of finitely generated varieties of commutative semigroups is a (not complete) sublattice of $\mathscr{L}(\mathrm{Com})$.

7.9. In [32] Sapir formulated ten basic questions concerning varieties, and considered them for varieties of semigroups. He demonstrated that, in general, varieties of semigroups have none of the nice properties suggested in his questions. To conclude our paper, we demonstrate that varieties of commutative semigroups enjoy all these properties. Below, we just state a suitable theorem and a corollary; for the very formulation of the questions the reader is referred to [32].

7.10. Recall that a variety is called small if its lattice of subvarieties is finite. If this variety is, in addition, finitely generated and finitely based, then it is called Cross.

Observe that for a sequence a of the form $(1,1, \ldots, 1)$ there are only finitely many filters $J \supseteq$ [a] , and therefore, in view of Examples 2 and 5 in $\S 6$, a variety $\mathscr{V}$ with $E(\mathscr{V})=E(J, m, r, \pi)$ and $\mathbf{a} \in J$ is small. We show that the converse holds, as well.

Indeed, given small $\mathscr{V}$, consider the sequence of equational theories $E_{n}=$ $E(\mathscr{V}) \vee\left\{x_{1} \cdots x_{n}=x_{1}^{r+1} x_{2} \cdots x_{n}\right\}^{*}, r=r(\mathscr{V})$. This sequence has to be finite; hence, $E_{n}=E_{n+1}$ for some $n \geq 1$. It follows that the identity $e: x_{1} \cdots x_{n}=$ $x_{1}^{r+1} x_{2} \cdots x_{n}$ is a consequence of $x_{1} \cdots x_{n+1}=x_{1}^{r+1} x_{2} \cdots x_{n+1}$ and the identities in $E(\mathscr{V})$. Applying Lemma 5.2, we infer easily that the identity $e$ is in $E(\mathscr{V})$, as required.

Note, that this argument holds for a variety with the ascending chain condition, as well.

It follows, in view of Theorem 7.7 , that every small variety is finitely generated, and whence, in view of Proposition 1.2, Cross.

On the other hand, Higgins [14] proved that a variety $\mathscr{V} \in \mathscr{L}(\mathrm{Com})$ is saturated in the sense of Howie and Isbell [16] (i.e., all epis are onto) if and only if a nontrivial identity of the form $x_{1} \cdots x_{n}=\left(x_{1} \cdots x_{n}\right)^{k}$ holds in $\mathscr{V}$.

Combining all these remarks we have the following.

7.11. Theorem. For a variety $\mathscr{V}$ of commutative semigroups the following conditions are equivalent. 
(i) $\mathscr{V}$ is Cross.

(ii) $\mathscr{V}$ is small.

(iii) The ascending chain condition holds for the subvarieties of $\mathscr{V}$.

(iv) $\mathscr{V}$ is saturated.

(v) A nontrivial identity of the form $x_{1} \cdots x_{n}=x_{1}^{\alpha_{1}} \cdots x_{n}^{\alpha_{n}}$ holds in $\mathscr{V}$ for some $n \geq 1$.

Following Sapir [32], call a sublattice of a lattice of varieties stable if it is closed under taking covers and subvarieties. A just non-Cross variety is a variety which is not Cross, but all of its proper subvarieties are Cross.

While there are infinitely many just non-Cross varieties of semigroups [32], the only known example of a just non-Cross variety of commutative semigroups is that of the variety $\mathscr{V}\left(x^{2}=0\right)$ defined by the law $x^{2}=0$ [35]. Using again arguments based on Lemma 5.2, we get easily the following.

7.12. Corollary. The commutative semigroup Cross varieties form a stable sublattice of $\mathscr{L}(\mathrm{Com})$; the variety $\mathscr{V}\left(x^{2}=0\right)$ is the only just non-Cross variety of commutative semigroups.

\section{REFERENCES}

1. A. Ja. Aizenštat, On varieties of semigroups having finite number of subvarieties, Algebraic Theory of Semigroups, Colloq. Math. Soc. János Bolyai, vol. 20, Amsterdam, New York, 1979, pp. 33-41.

2. J. Almeida, Some order properties of the lattice of varieties of commutative semigroups, Canad. J. Math. 38 (1986), 19-47.

3. J. Almeida and N. R. Reilly, Generalized varieties of commutative and nilpotent semigroups, Semigroup Forum 30 (1984), 77-98.

4. S. Burris and E. Nelson, Embedding $\Pi_{m}$ in the lattice of equational classes of commutative semigroups, Proc. Amer. Math. Soc. 30 (1971), 37-39.

5. J. Dudek and A. Kisielewicz, Totally commutative semigroups, J. Austral. Math. Soc. (Ser. A) 51 (1991), 381-399.

6. S. Eilenberg, Automata, languages, and machines, Vol. B, Academic Press, San Diego, 1976.

7. T. Evans, The lattice of semigroup varieties, Semigroup Forum 2 (1971), 1-43.

8. Some connections between residual finiteness, finite embeddability and the word problem, J. London Math. Soc. 1 (1969), 399-403.

9. __ Some solvable word problems, Word Problems II, North-Holland, Amsterdam, 1980, pp. 87-100.

10. M. R. Garey and D. S. Johnson, Computers and intractability, Freeman, New York, 1979.

11. J. A. Gerhard and M. Petrich, Varieties of bands revisited, Proc. London Math. Soc. (3) 58 (1989), 323-350.

12. G. Grätzer, Universal algebra, 2nd ed., Springer-Verlag, Berlin and New York, 1979.

13. T. J. Head, The varieties of commutative monoids, Nieuw Arch. Wisk. (4) 16 (1968), 203-206.

14. P. M. Higgins, The varieties of commutative semigroups for which epis are onto, Proc. Roy. Soc. Edinburgh Sect. A 94 (1983), 1-7.

15. G. Higman, Ordering by divisibility in abstract algebras, Proc. London Math. Soc. (3) 2 (1952), 326-336.

16. J. M. Howie and J. R. Isbell, Epimorphisms and dominions. II, J. Algebra 6 (1967), 7-21. 
17. A. Kisielewicz, All pseudovarieties of commutative semigroups, Semigroups with Applications (Oberwolfach, 1991) (J. M. Howie et al., eds.), World Scientific, Singapore, 1992, pp. 78-89.

18. I. O. Korjakov, $A$ sketch of the lattice of commutative nilpotent semigroup varieties, Semigroup Forum 24 (1982), 285-317.

19. J. Kruskal, The theory of well-quasi ordering: a frequently discovered concept, J. Combin. Theory Ser. A 13 (1972), 297-305.

20. G. Lallement, Semigroups and combinatorial applications, Wiley-Interscience, New York, 1979.

21. S. A. Malyšev, The lattice of subvarieties of the variety $\Pi\left(x_{1} \cdots x_{n}=x_{1} \cdots x_{n}^{k+1}, x y=y x\right)$, Semigroup Varieties and Semigroups of Endomorphisms, Leningrad. Gos. Ped. Inst., Leningrad, 1979, pp. 113-122. (Russian)

22. I. I. Me'lnik, The description of some lattices of semigroup varieties, Izv. Vyssh. Uchebn. Zaved. Mat. 7 (1972), 67-74. (Russian)

23. E. W. Mayr and A. R. Meyer, The complexity of the word problems for commutative semigroups and polynomial ideals, Adv. in Math. 46 (1982), 305-329.

24. E. Nelson, The lattice of equational classes of commutative semigroups, Canad. J. Math. 23 (1971), 875-895.

25. _ The lattice of equational classes of semigroups with zero, Canad. Math. Bull. 14 (1971), 531-534.

26. H. Neumann, Varieties of groups, Springer-Verlag, Berlin and New York, 1967.

27. P. Perkins, Bases for equational theories of semigroups, J. Algebra 11 (1969), 298-314.

28. M. Petrich, Lectures in semigroups, Wiley, London, 1977.

29. J. E. Pin, Varieties of formal languages, Plenum, London, 1986.

30. L. Polák, On varieties of completely regular semigroups. I, II, III, Semigroup Forum 32 (1985), 97-123; 36 (1987), 253-184; 37 (1988), 1-30.

31. P. Pudlák and J. Tůma, Every finite lattice can be embedded into a finite partition lattice, Algebra Universalis 10 (1980), 74-95.

32. M. V. Sapir, On Cross semigroup varieties and related problems, Semigroup Forum 42 (1991), 345-364.

33. O. B. Sapir, The axiomatic and the basis series of varieties of commutative semigroups, Ural. Gos. Univ. Mat. Zap. 14 (1988), no. 3, 112-119. (Russian)

34. L. N. Shevrin and E. V. Sukhanov, Structural aspects of the theory of varieties of semigroups, Soviet Math. (Iz. VUZ) 33 (1989), no. 6, 1-54.

35. L. N. Shevrin and M. V. Volkov, Identities of semigroups, Soviet Math. (Iz. VUZ) 29 (1985), no. 11, 1-64.

36. R. Schwabauer, A note on commutative semigroups, Proc. Amer. Math. Soc. 20 (1969), 503-504.

37. __ Commutative semigroup laws, Proc. Amer. Math. Soc. 22 (1969), 591-595.

38. M. A. Taitslin, Algorithmic problems for commutative semigroups, Soviet. Math. Dokl. 9 (1968), 201-204.

39. W. Taylor, Equational logic, Houston J. Math., 1979, Survey, 1-83.

40. B. M. Vernikov and M. V. Volkov, Lattices of nilpotent varieties of semigroups, Ural. Gos. Univ. Mat. Zap. 14 (1988), no. 3, 53-65. (Russian)

41. M. V. Volkov, Commutative semigroup varieties with distributive subvariety lattices, Contributions to General Algebra, vol. 7, Wien, Stuttgart, 1991, pp. 351-359.

42. __ Semigroup varieties with modular subvariety lattices, preprint.

Institute of Mathematics, Wroclaw University, Wroclaw, Poland

E-mail address: kisiel@math.impwr.wroc.edu.pl

Fachbereich Mathematik AG 1, Technische Hochschule Darmstadt, Darmstadt, GerMANY 